\title{
Application of Personalized Three-Dimensional Printing for Shoulder Joint Prosthesis in the Treatment of Shoulder Joint Tuberculosis: A Case Report
}

\author{
Yanlong Han \\ People's Hospital of Xinjiang Uygur Autonomous Region \\ Habaxi Kaken \\ people's hospital of xinjiang uygur autonomous region \\ Wei Zhao \\ People's Hospital of Xinjiang Uygur Autonomous Region \\ Ainiwaerjiang Damaola \\ People's Hospital of Xinjiang Uygur Autonomous Region \\ Li Wang ( $\nabla$ hanyanlong2017@163.com ) \\ People's Hospital of Xinjiang Uygur Autonomous Region
}

\section{Research article}

Keywords: shoulder joint, 3D printing, shoulder joint tuberculosis, reverse total shoulder arthroplasty

Posted Date: November 9th, 2020

DOI: https://doi.org/10.21203/rs.3.rs-102428/v1

License: (c) (i) This work is licensed under a Creative Commons Attribution 4.0 International License.

Read Full License 


\section{Abstract}

Background: This article presents an application of the use of a personalized three-dimensional (3D)printed reverse shoulder prosthesis to be used in a reverse total shoulder arthroplasty (RTSA) for a patient with tuberculosis of the shoulder joint.

Background: Patient concerns: A 37-year-old female, who suffered a trauma on her left shoulder 17 years ago, reported significant worsening pain and limited physical activity within the last 6 months. The left shoulder joint activity was clearly restricted. Diagnosis: Her clinical manifestations, bone changes in shoulder joint, and imaging results were all consistent with extended exposure to tuberculosis of the shoulder joint. Interventions: For this patient, a personalized 3D-printed titanium alloy shoulder joint prosthesis was used in the RTSA. The patient's stability was regularly monitored and followed up after operation. During pre-operation, post-operation, and follow-up, the scores of ASES, Neer Shoulder score, and Constant-Murley Shoulder were evaluated.

Results: 3D shoulder prosthesis was successfully implanted into the patient and complete anatomic reconstruction was performed intraoperatively. ASES, Neer, and Constant-Murley scores were gradually improved on follow-up. The X-ray image showed good bone healing and no changes in the position of the prosthesis seven and a half years post-operation. The functionality of the shoulder joint was well restored and no restrictions were found in activities of daily living.

Conclusion: Personalized 3D-printed shoulder joint prosthesis is very effective in the treatment of advanced tuberculosis of the shoulder joint. 3D-printed technology may be a novel and efficient method for similar arthroplasties and severe bone defects.

\section{Introduction}

Bone tuberculosis (TB) comes in two forms: TB of the joints and TB of the spine, both occurring in equal rates among patients. Joints affected include the hip, sacroiliac, knee, shoulder, elbow, and wrist joints. Out of all the ones mentioned previously, TB of the shoulder joint is a very rare disease ${ }^{1}$ with its morbidity ranging from $0.9 \%$ to $1.7 \%{ }^{2,3}$ Currently, there is no gold standard treatment for TB of the shoulder joint. But anti-TB drug therapy and surgery are the main methods that clinicians use today. Other traditional treatment methods such as arthrodesis, artificial shoulder arthroplasty, joint transplantation, and prosthesis allograft complex are not ideal treatment methods for shoulder reconstruction. ${ }^{4}$

The more effective method for treating end-stage shoulder joint diseases is through artificial shoulder arthroplasty ${ }^{5}$. However, current studies indicate the correction rate of shoulder arthroplasty to be approximately only $5 \%$ to $29 \% .{ }^{6-9}$ Common causes of injury include joint infection and instability, periprosthetic fractures, prosthesis loosening, and sports injury. ${ }^{10-15}$

Bone defect plays the most difficult problem for shoulder joint replacement. Additionally, the anatomic structure of the shoulder joint is relatively complex due to the shoulder rotator cuff requiring tendinous 
insertions and soft tissue relaxation. The shoulder joint is also at great risk for instability and dislocation during shoulder joint replacement. Currently, the medical technology of three-dimensional (3D) printing is currently being examined to provide new methods in solving bone defects and instability of artificial prosthesis. In this study, we report a patient who underwent a personalized 3D-printed reverse shoulder prosthesis for TB of the shoulder joint, with short-term follow-up used to evaluate the early efficacy and safety of the prosthesis.

\section{Case Presentation}

Before the study was conducted, informed consent was obtained from the patient, requesting to publish case details and images. The Ethics Committee of the People's Hospital of Xinjiang Uygur Autonomous Region approved this case study.

A 37-year-old female, who suffered from continuous pain on her left shoulder due to trauma seventeen years ago, complained of worsening pain and limited activity that began six months prior to admission. There was not pathology. Her x-ray (Fig. 1), computed tomography (CT) (Fig. 2), and magnetic resonance imaging (MRI) (Fig. 3) revealed a thickened acromion and the wall of the slip capsule of her left shoulder joint. T1 and T2 signal lesions in MRI were found in the soft tissue of the lower posterior region of the shoulder joint, with uneven and obvious enhancement around the sustained central necrosis. The smoothness of the articular surface appeared reduced and the articular space was narrow. The scores of American Shoulder and Elbow Surgeons' Form (ASES), Neer and Constant-Murley were 39, 37, and 40, respectively.

\section{Diagnosis}

Following admission, the patient underwent puncture biopsy of left the cervical lymph node. The pathological examination results showed coagulation necrosis of her left cervical lymph node accompanied by epithelioid granulomatous inflammation and multi-nucleated giant cell reaction. Specific infection and TB were considered. Upon takin the T-SPOT.TB test, she tested positive, leading to the patient's diagnosis as TB of the shoulder joint. Antituberculosis therapy was given first. Surgery was given after 6 months.

\section{Design of individual 3D-printed prosthesis}

Preoperative left upper limb x-ray and bilateral upper limb CT were done. Software MIMICS 19.0 was used to reconstruct data, after which it was exported in standard triangular language (STL) format. The STL file was imported into omagics 19.0 to make the support structure and then exported to the magics format of the 3D printing equipment (stereo lithography(sla-450,Shining,China)) to start printing. 3D-printed bone models were used to help surgeons assess bone defects, select prostheses, and design surgical procedures. The guide plate was printed with nylon powder and reverse shoulder joint prosthesis were printed with titanium alloy by 3D printing equipment(Fig. 4). 


\section{Surgical technique}

After the general anesthesia took effect, the patient was placed in a lateral position. After routine disinfection, an incision of approximately $25 \mathrm{~cm}$ was made in the deltoid, pectoralis major, and posterior scapularis muscles. The skin and subcutaneous tissues were incised layer by layer, including the fascia, and the deltoid and pectoralis major muscle space were entered. The deltoid and pectoralis major muscles were retracted to expose the rotator cuff around the shoulder joint and locate the greater tubercle and the internodal groove. The anterior joint capsule was opened to expose the rotary arm and the humeral head was then rotated out of the joint cavity. The humeral head was collapsed flat, necrosis was present, and serious destruction of the glenoid was observed. A large amount of scar granulation tissue filled the joint cavity, which were completely removed, as well as any dead bone. The greater tuberosity was positioned in the upper region of the greater tuberosity and was perforated. The reamer was placed in the medullary cavity and the positioning rod was installed. After adjusting for height and angle, the humeral head was removed with a swinging saw. The head handle mold test was installed, and the matching degree was observed. The glenoid center was positioned and measured according to the 3D printing test mold and the hole was positioned according to the guide plate. After confirmation, the prosthesis was installed and the screw back was fixed, completing the glenoid prosthesis process. The humerus cavity was then flushed and cemented, and the humerus prosthesis handle was installed. After the bone cement solidified, the shoulder joint was repositioned and moved with satisfactory results. The incision was sutured according to the level of anatomy. The left upper limb was suspended and fixed (Fig. 5).

\section{Surgical efficacy}

The personalized customized 3D-printed shoulder prosthesis was highly consistent with bone defects and the anatomical reconstruction of shoulder joint was successful. The soft tissues around the shoulder joint were successfully sewn into the prosthesis. The x-ray image done one day postoperative already showed that the prosthesis was in accurate position (Fig. 6). The surgical incision healed well, and postoperative complications (infection, fat liquefaction, and other postoperative complications) did not occur after surgery. The x-ray of the left shoulder six months and a year and a half post-operatively showed that the position and stability of prosthesis was still considerably satisfactory. ASES, Neer, and Constant-Murley score after three months were 78,85 , and 79 , respectively, and the scores a year and a half after surgery were 86,80 and 88 , respectively.

During the follow-up period, short-term complications such as infection of prosthetic implants, prosthesis loosening, and periprosthetic femoral fracture did not occur. The post-operative x-ray a year and a half later showed that the position of the prosthesis was still accurate (Fig. 7). The range of motion (ROM) of the shoulder joint was checked as well and yielded the following results: forward flexion $90^{\circ}$, backward extension $40^{\circ}$, abduction $80^{\circ}$, adduction $15^{\circ}$, external rotation $40^{\circ}$, and internal rotation $60^{\circ}$. At this time, the left arm was able to resume activities of daily living such as eating, writing, driving, lifting weights, 
and combing hair, with ease (Fig. 8). The function of shoulder joint was gradually well-restored, and the quality of daily life was significantly improved.

\section{Discussion}

This paper reported a patient diagnosed with TB of the shoulder joint, a rare articular disease, who underwent trans-shoulder replacement with a personalized 3D-printed shoulder prosthesis. Healthy shoulder joint anatomy and functional characteristics include rich muscle around the joint, good blood supply, wide ROM, and small contact area between the articular pelvis and humeral head, making the shoulder joint tuberculosis become exudate-less and abscess not that obvious. Muscle atrophy, which occurs because of continued immobilization, can affect the squareness between the two shoulders, cause dislocation, and induce poor joint function - which affects the activities of daily living and work. Joint function can be spared if joint TB can be treated early. If the disease is progresses further, TB can penetrate the joint surface and enter the joint cavity, making the soft bone surface of the joint suffer damage of varying degrees, and causing TB of the whole region. This patient developed left shoulder swelling and pain seventeen years ago, which became diseased due to the long duration of exposure to joint TB. She had to undergo shoulder replacement, but the anatomical structure of shoulder joint cannot be reconstructed well by traditional shoulder prosthesis. Loosening of the prosthesis, periprosthetic fracture, or even loss of bone mass can occur after traditional shoulder joint replacement, which may lead to disability in the patient. Therefore, appropriate shoulder prosthesis is significantly critical for successful shoulder replacement.

Currently, 3D-printing technology is developing rapidly in medical applications, especially in the clinical treatment of bone defects and other orthopedic diseases. ${ }^{16-20}$ According to the anatomical structure of the patient's skeleton and the characteristics of the disease itself, 3D-printing technology can now customize personalized prostheses or surgical instruments, building compatibility. Restoring the normal anatomy of the glenohumeral joint as much as possible is the key to achieving good function. The correct implant placement is the basis for balancing the tension of the surrounding soft tissue and maintaining the stability of the shoulder joint. For infectious arthritis of the shoulder, total shoulder arthroplasty is a good way to relieve pain and improve function

Some studies have indicated that 3D-printing porous prostheses are more effective in bone induction and soft tissue in-growth. ${ }^{13,21-23}$ The pore diameter is in the range of $682 \mathrm{~mm}-700 \mathrm{~mm}$, and the porosity rate of EBM-3D printing prosthesis is $51 \%-73 \%$, making it most favorable for bone penetration. ${ }^{24-25}$ Biocompatibility is an added advantage of 3D-printing materials. In addition, 3D-printing technology can solve some problems with the surgical prosthesis design of the clavicle, pelvis, spine, femur, and radius fractures as well as preoperative diagnosis. ${ }^{26-27}$

This technology can not only help surgeons assess bone damage more directly and clearly, but it can also reduce surgery time, anesthesia risk, and blood loss. Based on the aforementioned advantages, personalized 3D-printed shoulder joint prosthesis was the best option for this patient, and the 3D-printed 
reverse shoulder joint model was designed according to the imaging materials of the patient before surgery. The personalized 3D-printed prostheses were implanted into this patient based on accurate preoperative design, appropriate position and size, and passive shoulder recovery. Meanwhile, the surrounding tendon and other tissues were repaired with a patch on the pre-designed hole for establishing further anatomical fixation of the prosthesis. This was done so that the prosthesis would not loosen or fracture after surgery. The shoulder ROM as well as the relevant scores improved, showing that the shortterm clinical effect of personalized 3D-printing prosthesis after surgery was highly satisfactory.

Personalized 3D-printing technology could be used to resect shoulder joint disease, enable safe shoulder joint replacement and revision surgery, and repair bone defects and reconstruction of the pelvic cavity after pelvic tumor resection. ${ }^{28,29}$ Furthermore, this will be the future trend of medical development, which may be applied to multiple areas of orthopedics such as complex bone defects, special bone disease, and anatomical structures. Traditional treatment method of bone defects includes cement implants or autologous bone transplantations. Now, 3D-printing technology provides a more effective method that avoids further bone damage and enables better performing anatomical and functional reconstruction. 3D-printed prosthesis provides suitable high precision repair. For total knee total hip arthroplasty, soft tissue conditions of some patients are poor, and traditional prostheses cannot be fixed firmly. However, 3D-printing technology can help design personalized screws and soft tissue in-growth holes to improve the stability of the prosthesis.

But it is undeniable that 3D printing technology still faces many challenges, and there are certain limitations, which prohibit its wide application. Currently, the 3D printing manufacturing process takes a long time and is not suitable for many first-aid cases ${ }^{30}$. Fortunately, with the continuous improvement of 3D printing technology, personalized 3D printing prosthesis can be designed and produced within seven ten days. However, personalized 3D printed prostheses are expensive and not all patients can afford them ${ }^{31}$. We believe that with the rapid development of productivity and digital technology, 3D printing technology will become cheaper and more common in the next decade.

The results of short-term follow-up were satisfactory, but long-term follow-up was not carried out. The ROM of the shoulder joint and the patient's quality of life were significantly improved during follow-up. At present, 3D-printing technology is still a new modality for orthopedic treatment, but will certainly play a significant and more common role in the field as its gradual use increases in clinical practice. Custommade prosthetics, however, cannot be mass-produced as they are very expensive to make. Therefore, we believe that personalized 3D-printed prosthesis can used for complex situations where traditional prosthesis cannot be recommended. At present, the clinical application of 3D-printing technology has not been fully-confirmed by clinical evidence. Therefore, surgeons should exercise caution and treat the technology rationally and sparingly, when applicable. A future direction for this treatment must include large amounts of clinical data to confirm the long-term clinical effects of personalized 3D-printed prostheses.

\section{Conclusion}


The application of personalized 3D-printed reverse shoulder prosthesis in the RTSA achieves short-term satisfactory clinical results. It provides a novel method for complex arthroplasty or revision arthroplasty procedures with similar severe bone defects. However, the long-term follow-up as well as a large amount of clinical evidence are still required to confirm the reliability of the treatment.

\section{Abbreviations}

\begin{tabular}{|ll|}
\hline CT & computed tomography \\
\hline 3D & three-dimensional \\
\hline ROM & range of motion \\
\hline STL & standard triangular language \\
\hline VAS & Visual Analog Scale \\
\hline JSSF & Japanese Society for Surgery of the Foot \\
\hline ASES & American Shoulder and Elbow Surgeons' Form \\
\hline MRI & magnetic resonance imaging \\
\hline RTSA & reverse total shoulder arthroplasty \\
\hline TB & tuberculosis \\
\hline
\end{tabular}

\section{Declarations}

Before the study was conducted, informed consent was obtained from the patient, requesting to publish case details and images. The Ethics Committee of the People's Hospital of Xinjiang Uygur Autonomous Region approved this case study.

Consent for publication: All authors agree to be published

Availability of data and materials: All of data and materials are authentic.

Conflict of Interest: The authors declare that they have no conflicts of interest.

Funding Requests: This study did not receive any funding.

\section{Author contributions}

Conceptualization: Li Wang.

Data curation: Yan-Long Han

Formal analysis: Li Wang, Yanlong Han 
Investigation: Habaxi·Kaken, Ainiwaerjiang·Damaola.

Methodology: Li Wang, Yanlong Han.

Project administration: Li Wang, Yanlong Han.

Resources: Habaxi·Kaken, Wei Zhao.

Software: Li Wang, Yanlong Han

Supervision: Li Wang.

Validation: Li Wang.

Writing - original draft: Li Wang, Yanlong Han.

Writing - review \& editing: Li Wang, Yanlong Han

\section{Acknowledgements}

Thanks to all the authors who contributed to this article.

\section{References}

[1] Deshmukh A, Deo S, Salgia AK, Agarwal T, A Rare Unusual Case Presentation of the Tuberculosis of the Shoulder Joint. J Orthop Case Rep.2013 Oct-Dec;3(4):23-5.

[2] Tuli S. M. Tuberculosis of the skeletal system. 2nd ed. New Delhi, Jaypee Brothers Medical Publisher (P) Ltd. 1993.

[3] Sankaran B. Tuberculosis Of Bones \& Joints; Ind. J. Tub., 1993, 40,109.

[4] Tang X, Guo W, Yang R, et al. Synthetic mesh improves shoulder function after intraarticular resection and prosthetic replacement of proximal humerus. Clin Orthop Relat Res 2015;473:1464-71.

[5] Werner BC, Burrus MT, Begho I, et al. Early revision within 1 year after shoulder arthroplasty: patient factors and etiology. J Shoulder Elbow Surg/Am Shoulder Elbow Surg 2015;24:323-30.

[6] Voorde PC, Rasmussen JV, Olsen BS, et al. Resurfacing shoulder arthroplasty for the treatment of severe rheumatoid arthritis: outcome in 167 patients from the Danish Shoulder Registry. Acta Orthop $2015 ; 86: 293-7$.

[7] Vuillermin CB, Trump ME, Barwood SA, et al. Catastrophic failure of a low profile metal-backed glenoid component after total shoulder arthroplasty. Int J Shoulder Surg 2015;9:121-7. 
[8] Tanagho A, Morgan B, Ravenscroft M. Total humeral endoprosthesis replacement to salvage periprosthetic fractures in rheumatoid arthritis. Orthopedics 2017;40:e363-6.

[9] Roberson TA, Bentley JC, Griscom JT, et al. Outcomes of total shoulder arthroplasty in patients younger than 65 years: a systematic review. J Shoulder Elbow Surg 2017;26:1298-306.

[10] Fevang BTS, Lie SA, Havelin LI, et al. Risk factors for revision after shoulder arthroplasty. Acta Orthop 2009;80:83-91.

[11] Linberg CJ, Sperling JW, Schleck CD, et al. Shoulder arthroplasty in morbidly obese patients. J Shoulder Elbow Surg 2009;18:903-6.

[12] Farng E, Zingmond D, Krenek L, et al. Factors predicting complication rates after primary shoulder arthroplasty. J Shoulder Elbow Surg 2011;20:557-63.

[13] Singh JA, Sperling JW, Cofield RH. Revision surgery following Total Shoulder Arthroplasty: Analysis of 2,588 shoulders over 3 decades (1976-2008). J Bone Jt Surg Br Vol 2011;93:1513-7.

[14] Rasmussen JV. Outcome and risk of revision following shoulder replacement in patients with glenohumeral osteoarthritis. Acta Orthop Suppl 2014;85:1-23.

[15] Rasmussen JV, Polk A, Brorson S, et al. Patient-reported outcome and risk of revision after shoulder replacement for osteoarthritis. Acta Orthop 2014;85:117-22.

[16] Rengier F, Mehndiratta A, von Tengg-Kobligk H, et al. 3D printing based on imaging data: review of medical applications. Int J Comput Assist Radiol Surg 2010;5:335-41.

[17] Li H, Wang L, Mao Y, et al. Revision of complex acetabular defects using cages with the aid of rapid prototyping. J Arthroplasty 2013;28:1770-5.

[18] Tan $\mathrm{H}$, Yang K, Wei P, et al. A novel preoperative planning technique using a combination of ct angiography and three-dimensional printing for complex toe-to-hand reconstruction. J Reconstr Microsurg 2015;31:369-77.

[19] Wang D, Wang $Y$, Wang J, et al. Design and fabrication of a precision template for spine surgery using selective laser melting (SLM). Materials (Basel) 2016;22: pii:E608.

[20] Iqbal T, Shi L, Wang L, et al. Development of finite element model for customized prostheses design for patient with pelvic bone tumor. Proc Inst Mech Eng H 2017;231:525-33.

[21] OtawaN, Sumida T, Kitagaki $H$, et al. Custom-made titaniumdevices as membranes for bone augmentation in implant treatment: Modeling accuracy of titanium products constructed with selective laser melting. J Craniomaxillofac Surg 2015;43:1289-95. 
[22] Qian C, Zhang F, Sun J. Fabrication of Ti/HA composite and functionally graded implant by threedimensional printing. Biomed Mater Eng 2015;25:127-36.

[23] Shao $\mathrm{H}, \mathrm{Ke} X$, Liu A, et al. Bone regeneration in 3D printing bioactive ceramic scaffolds with improved tissue/material interface pore architecture in thin-wall bone defect. Biofabrication 2017;9:025003.

[24] Lv J, Jia Z, Li J, et al. Electron beam melting fabrication of porous ti6al4v scaffolds: cytocompatibility and osteogenesis. Adv Eng Mater 2015;

\section{$17: 1391-8$}

[25] Markhoff J,Wieding J, Weissmann V, et al. Influence of different three-dimensional open porous titanium scaffold designs on human osteoblasts behavior in static and dynamic cell investigations. Materials 2015;8:5490-507.

[26] Kim HN, Xiao NL, Noh KC. Use of a real-size 3D-printed model as a preoperative and intraoperative tool for minimally invasive plating of comminuted midshaft clavicle fractures. J Orthop Surg Res 2015;10:91.

[27] Wu XB, Wang JQ, Zhao CP, et al. Printed three-dimensional anatomic templates for virtual preoperative planning before reconstruction of old pelvic injuries: initial results. Chin Med J (Engl) 2015;128:477-82.

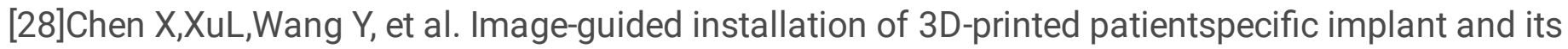
application in pelvic tumor resection and reconstruction surgery. Comput Methods Programs Biomed 2016;125:66-78.

[29] Shan XF, Chen HM, Liang J, et al. Surgical reconstruction of maxillary and mandibular defects using a printed titanium mesh. JOral Maxillofac Surg 2015;73:e1437-1439.

[30] ByrneN,Velasco ForteM,Tandon A,Valverde I, HussainT.Asystematic review of image segmentation methodology, used in the additive manufacture of patient-specific 3Dprintedmodels of the cardiovascular system. JRSM Cardiovasc Dis. 2016;5:2048004016645467.

[31] Riggs KW, Dsouza G, Broderick JT, Moore RA, Morales DLS. 3D-printed models optimize preoperative planning for pediatric cardiac tumor debulking. Transl Pediatr. 2018;7(3):196-202.

\section{Figures}



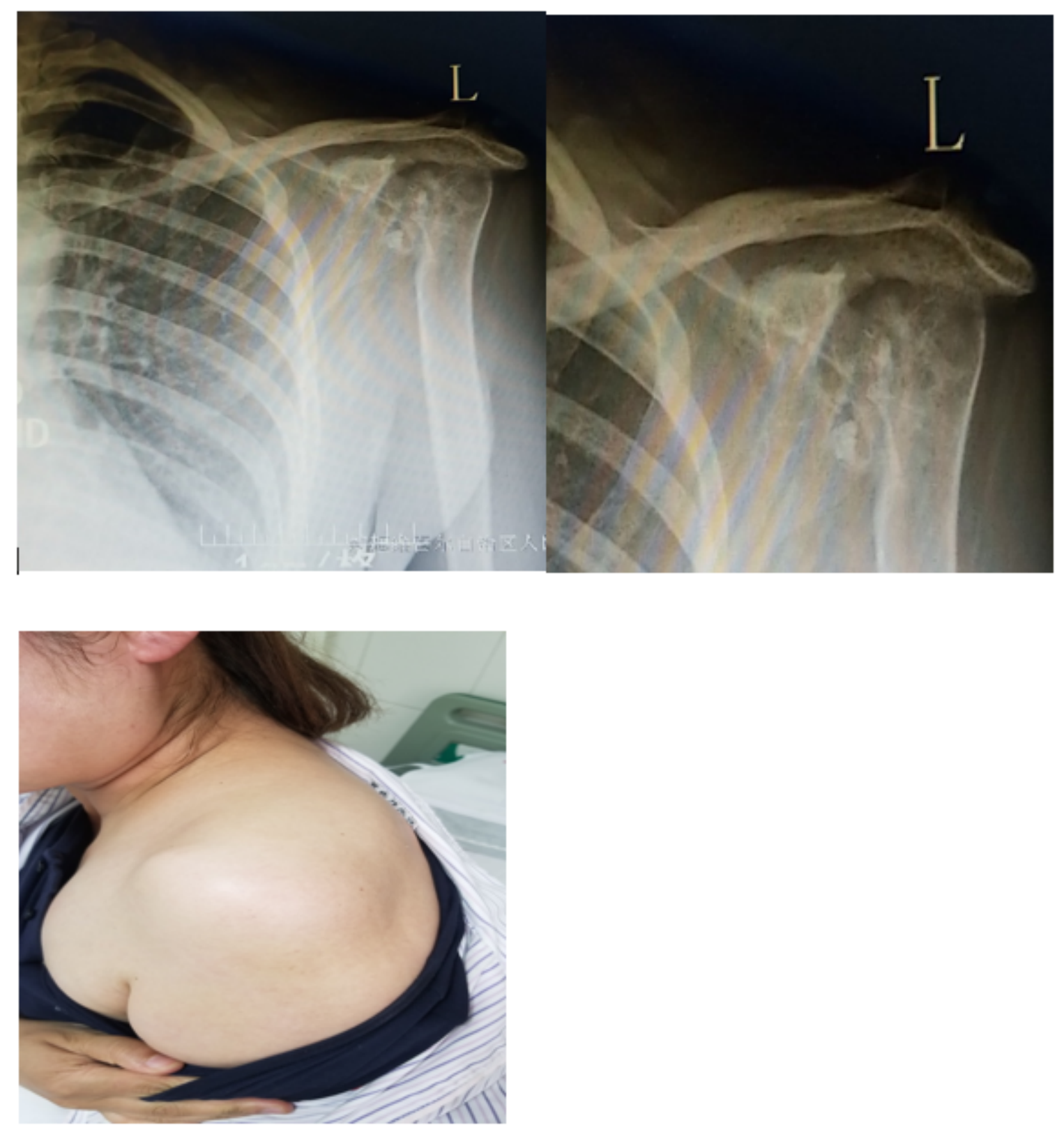

Figure 1

Preoperative x-ray and shoulder image. Limitation of shoulder joint activity may be seen. 

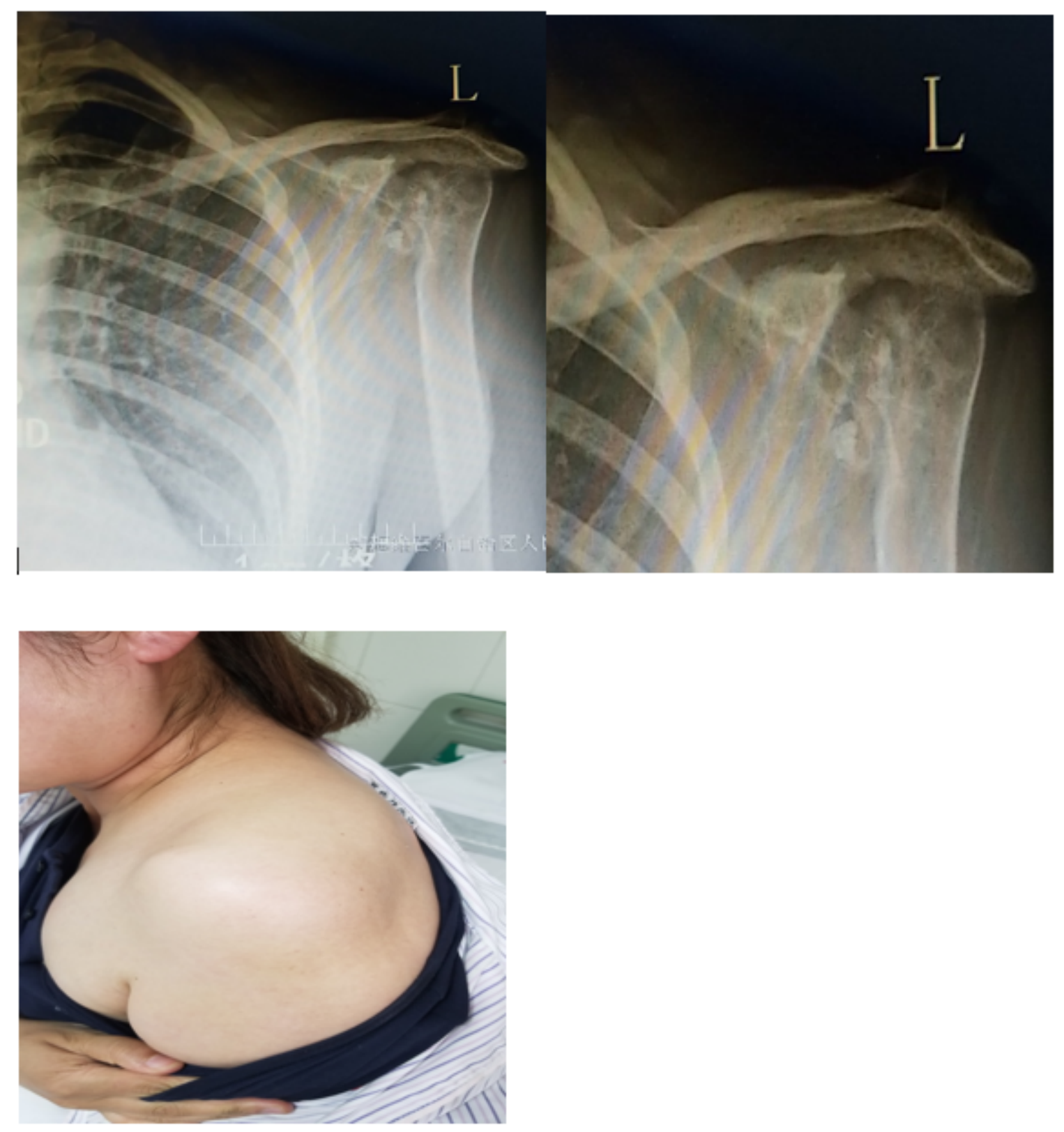

Figure 1

Preoperative x-ray and shoulder image. Limitation of shoulder joint activity may be seen. 

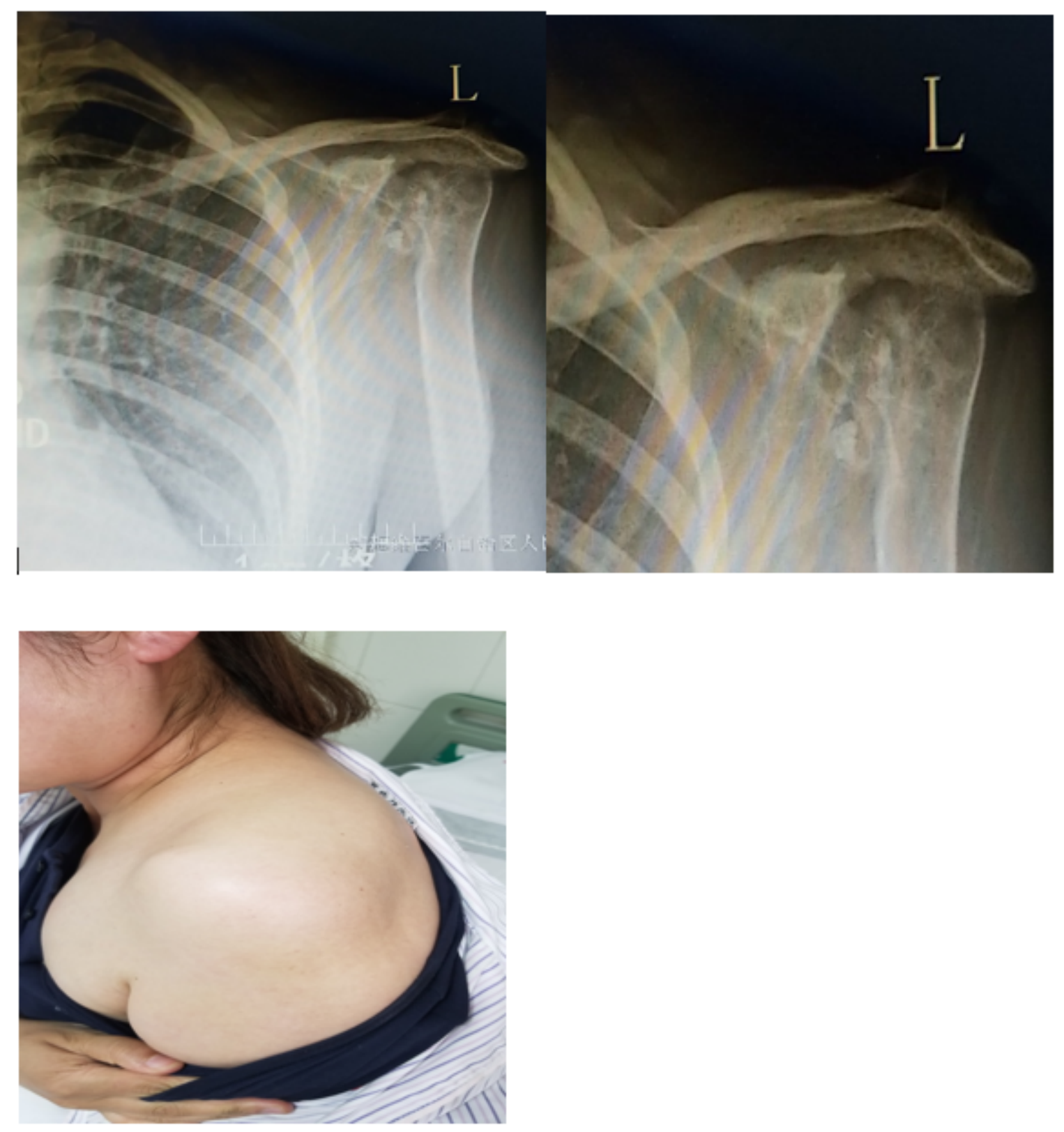

Figure 1

Preoperative x-ray and shoulder image. Limitation of shoulder joint activity may be seen. 

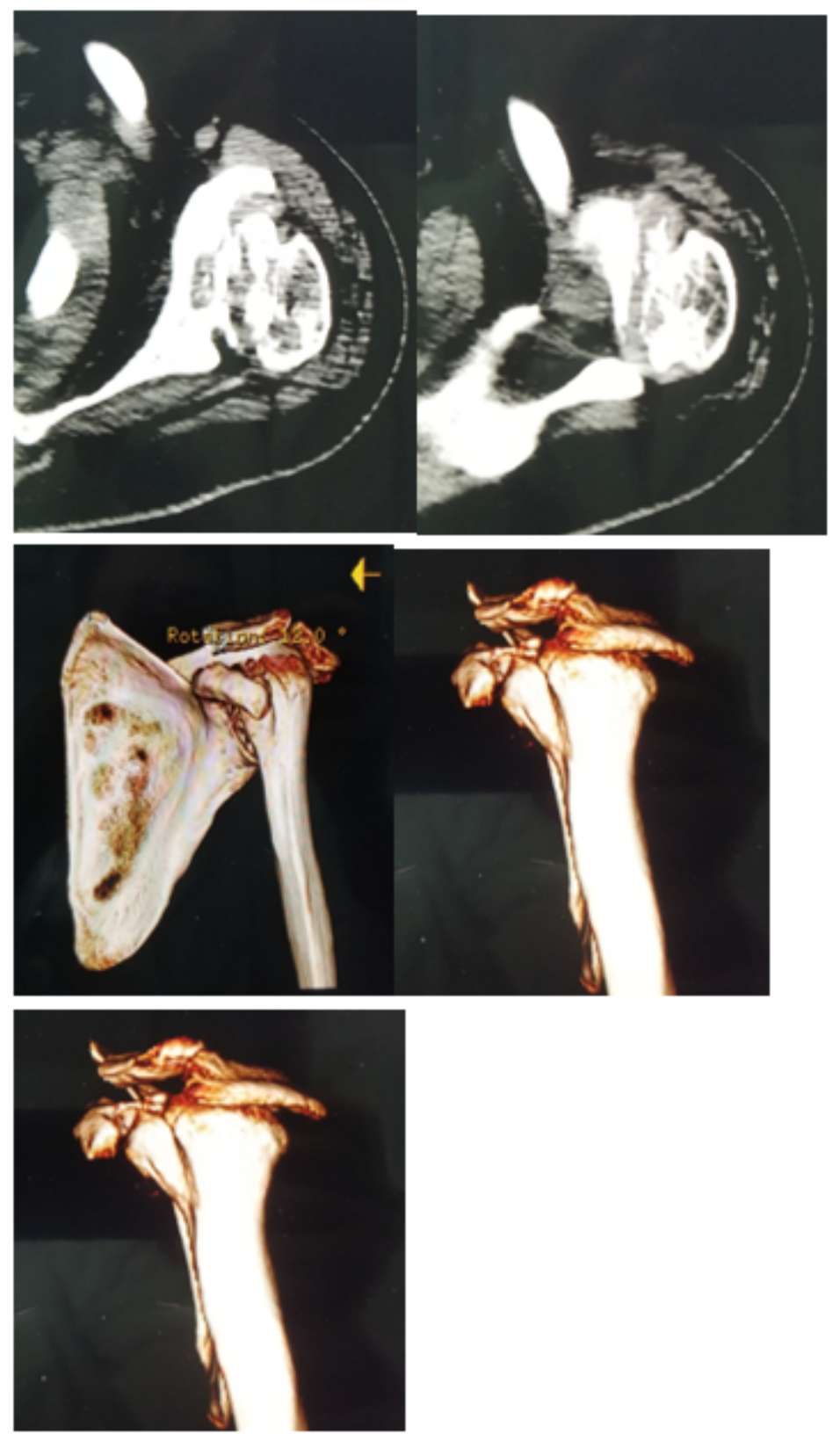

Figure 2

Preoperative CT indicated narrowing of shoulder joint space, humeral head deformation and bone destruction, accompanied by scattered bone formation 

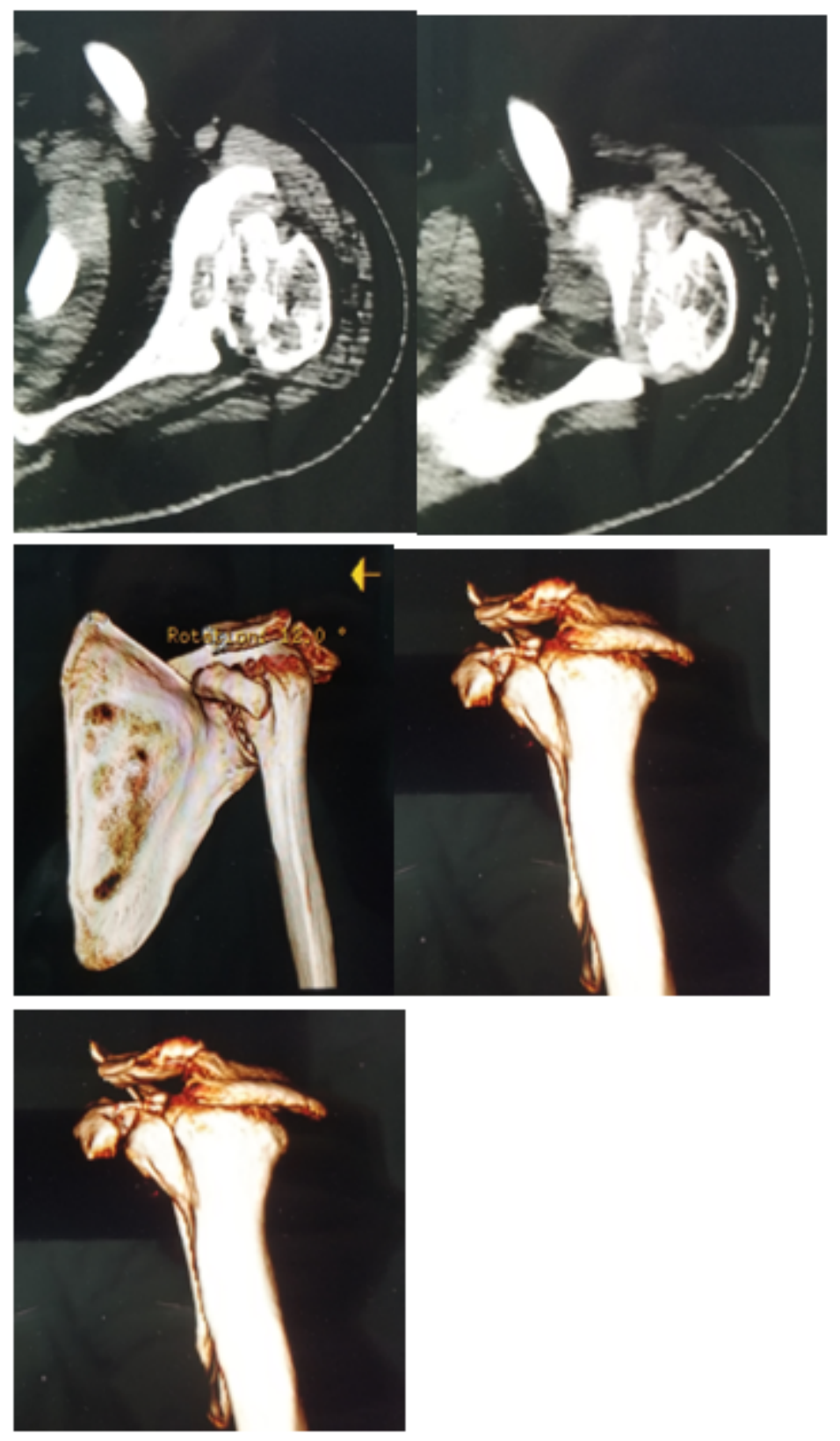

Figure 2

Preoperative CT indicated narrowing of shoulder joint space, humeral head deformation and bone destruction, accompanied by scattered bone formation 

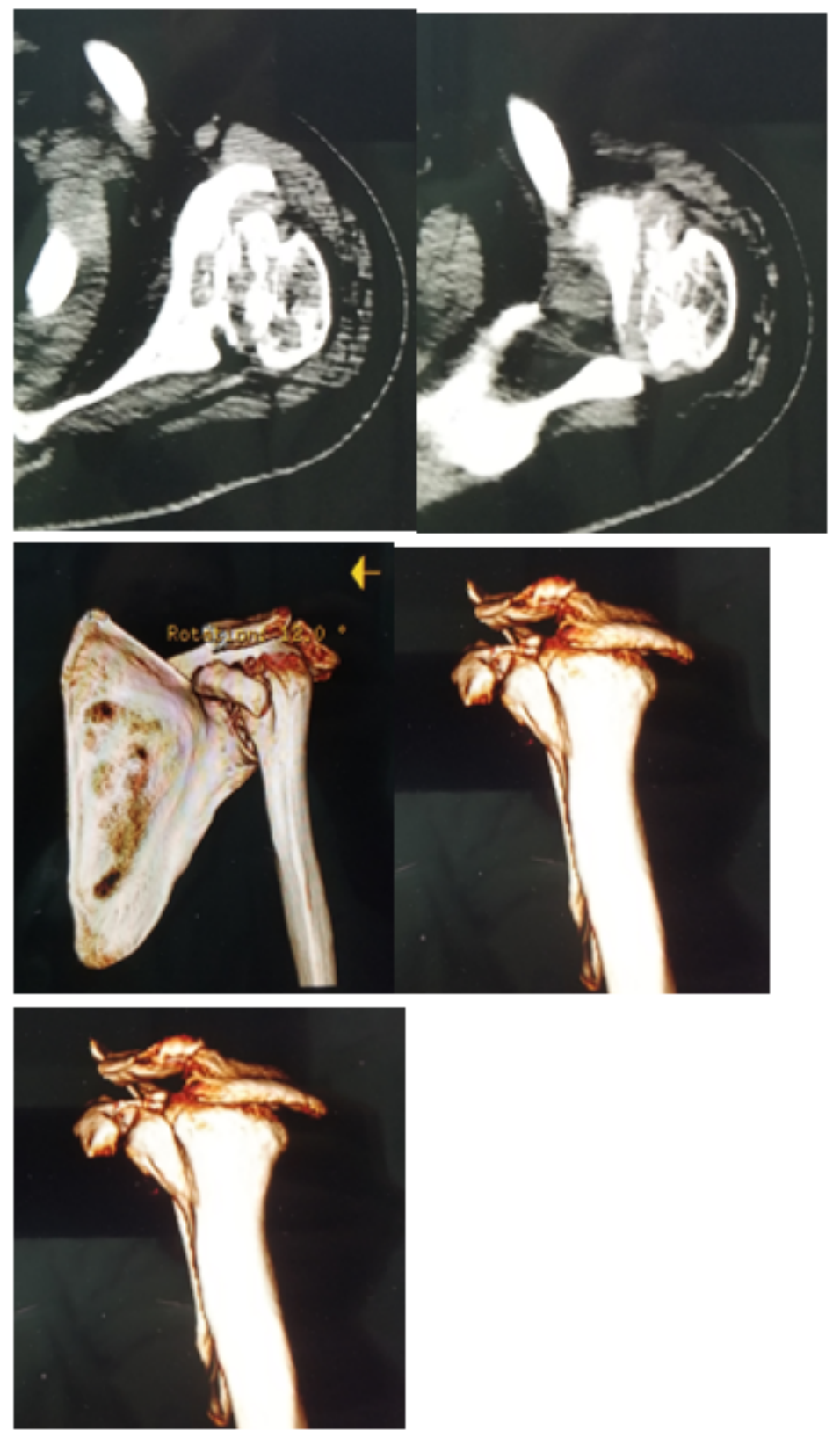

Figure 2

Preoperative CT indicated narrowing of shoulder joint space, humeral head deformation and bone destruction, accompanied by scattered bone formation 


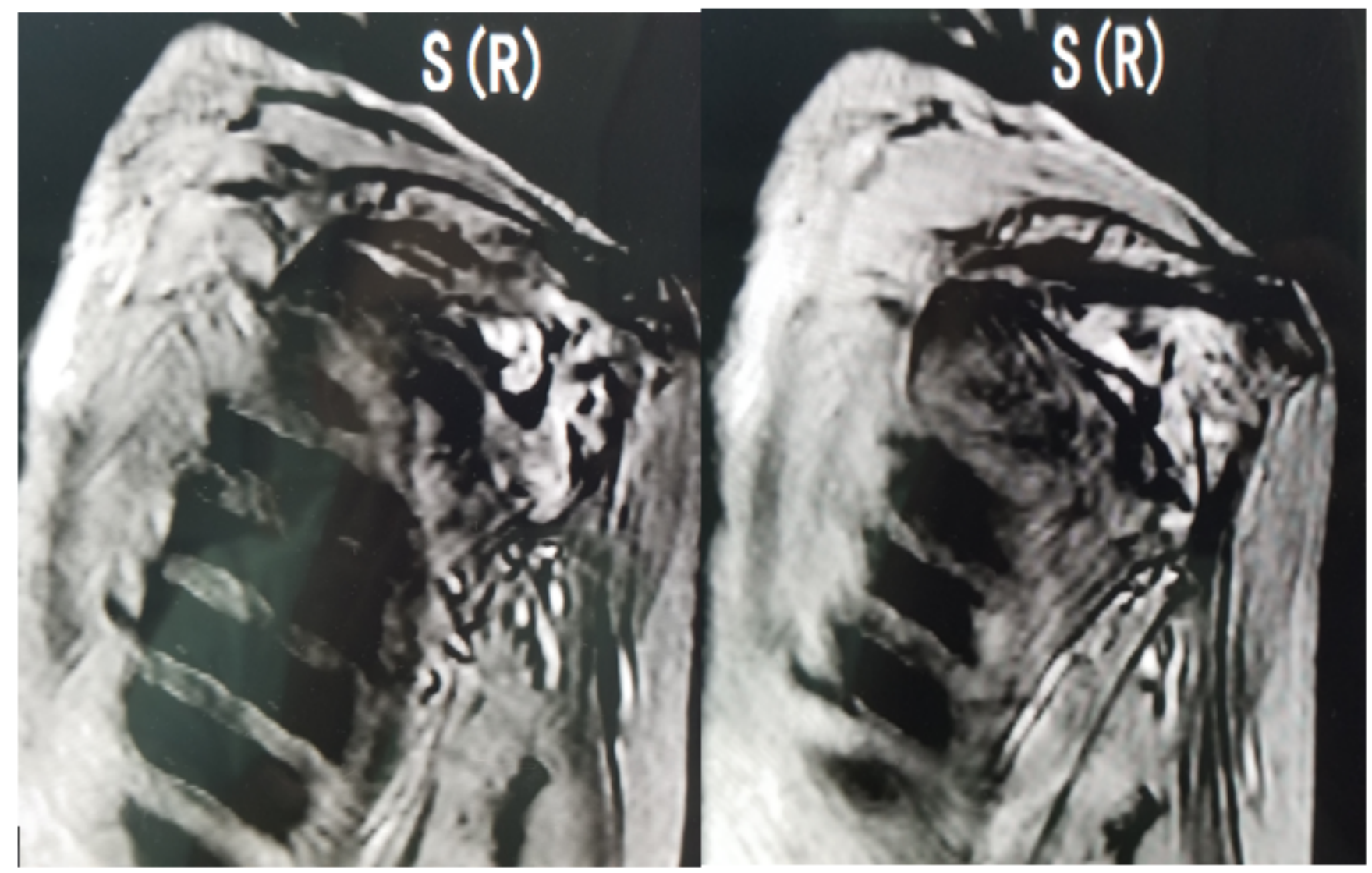

Figure 3

Preoperative MRI indicated narrowing of shoulder joint space, humeral head deformation, abnormal signals of internal bone destruction, and abnormal signals of swelling level in surrounding soft tissue 


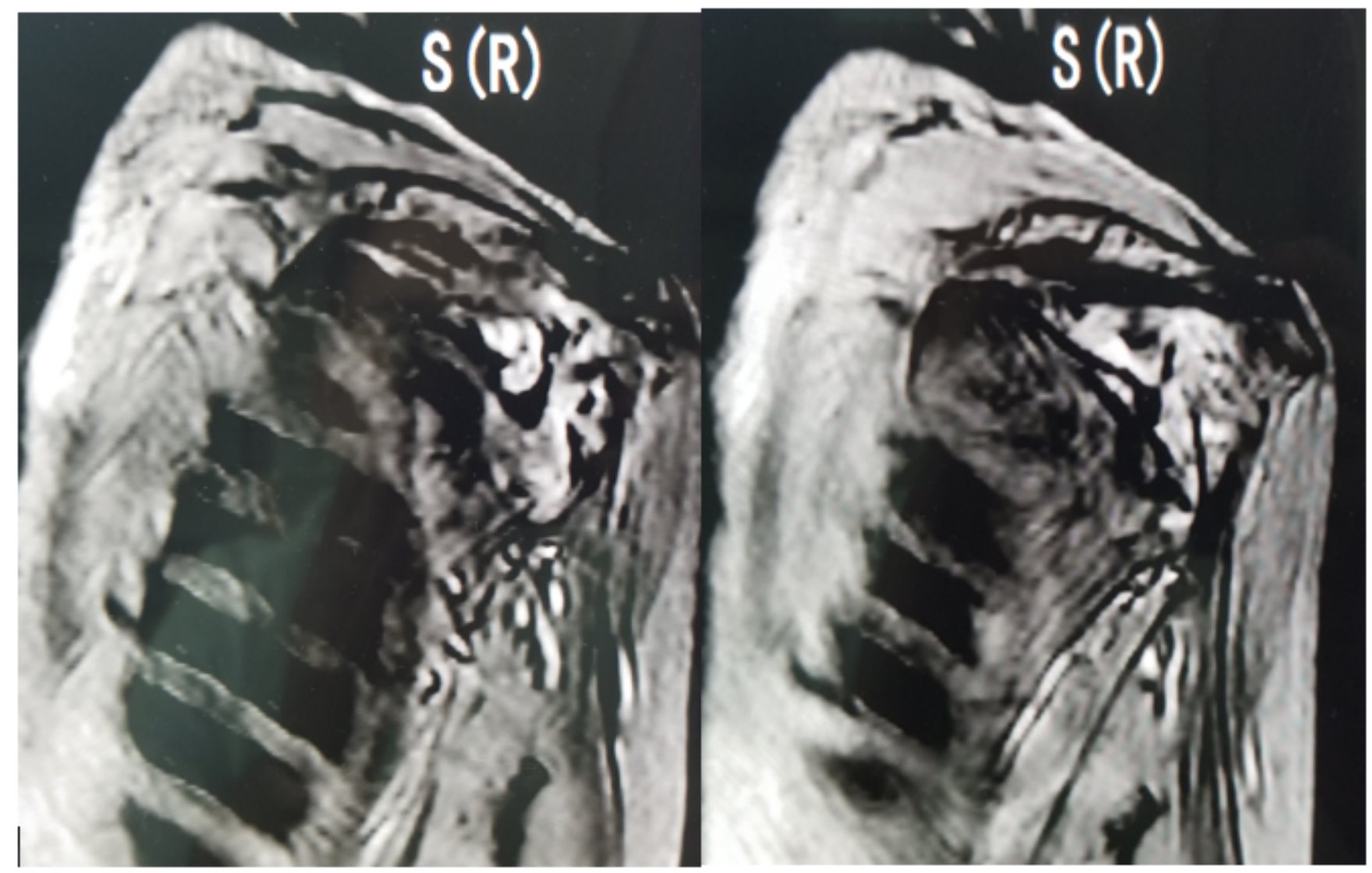

Figure 3

Preoperative MRI indicated narrowing of shoulder joint space, humeral head deformation, abnormal signals of internal bone destruction, and abnormal signals of swelling level in surrounding soft tissue 


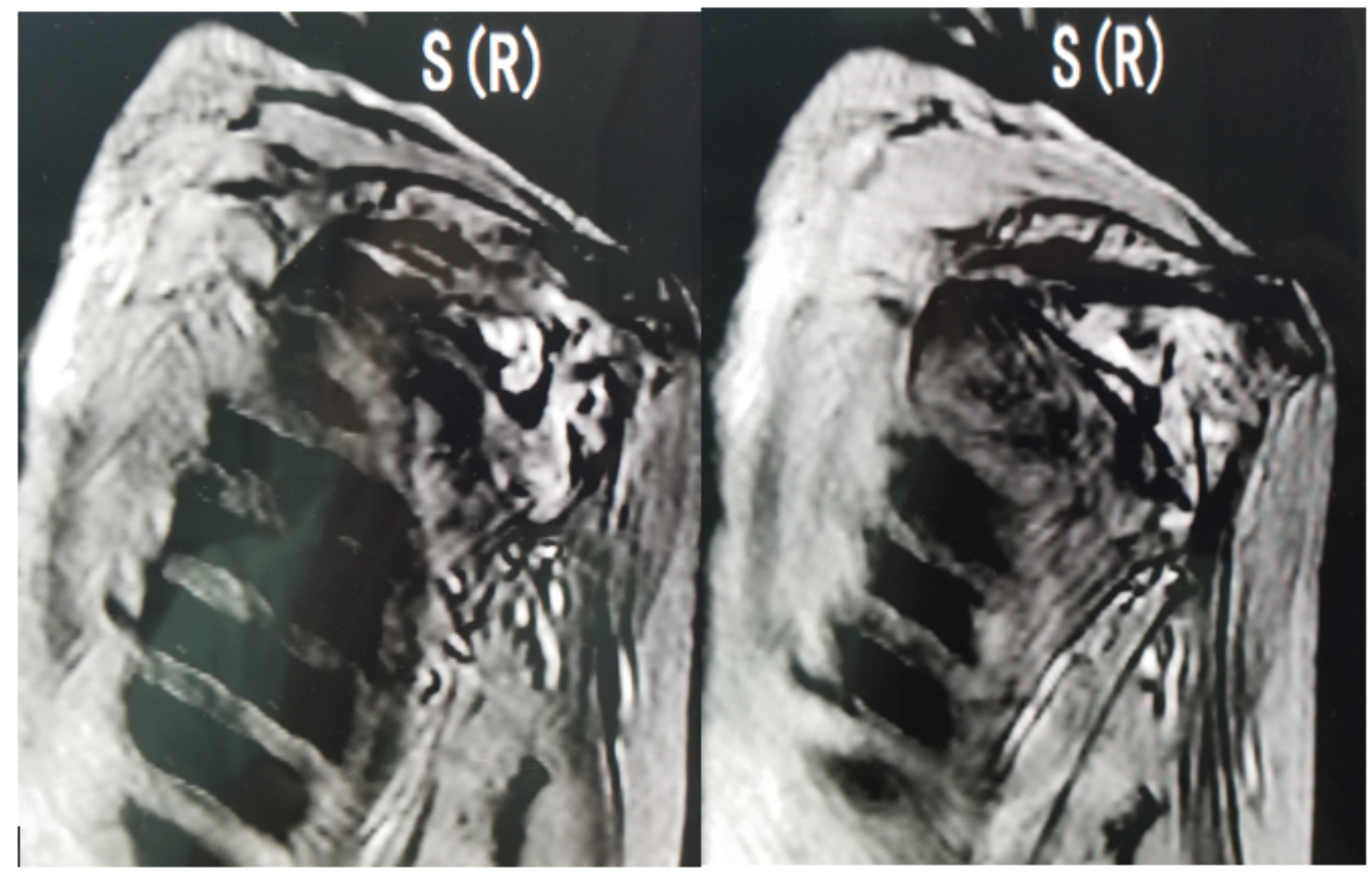

Figure 3

Preoperative MRI indicated narrowing of shoulder joint space, humeral head deformation, abnormal signals of internal bone destruction, and abnormal signals of swelling level in surrounding soft tissue 

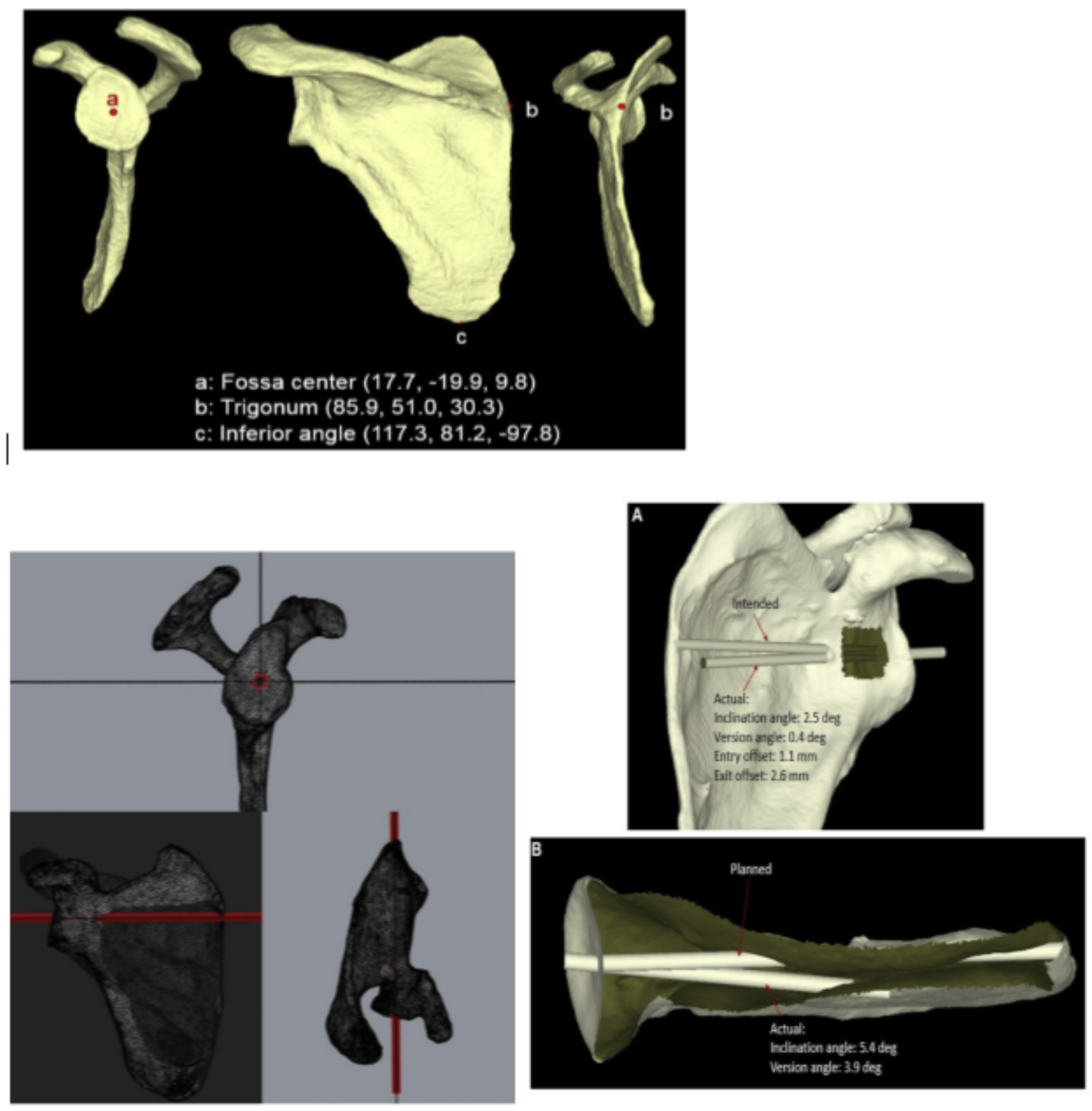

\section{Figure 4}

The STL file was imported into omagics 19.0 to make the support structure and then exported to the magics format of the 3D printing equipment (stereo lithography \sla-450,Shining,China)) to start printing. 

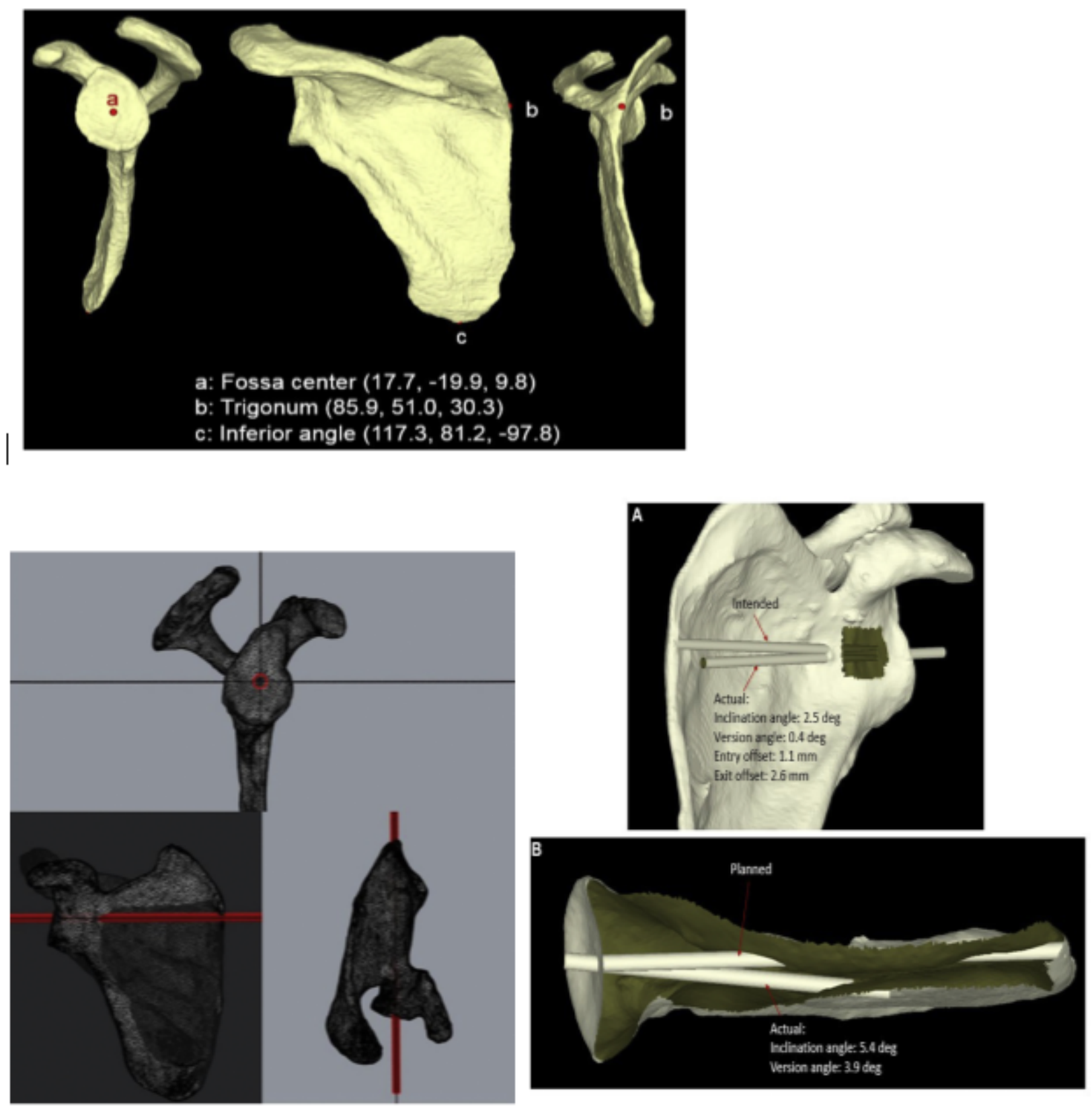

\section{Figure 4}

The STL file was imported into omagics 19.0 to make the support structure and then exported to the magics format of the 3D printing equipment (stereo lithography \sla-450,Shining,China)) to start printing. 


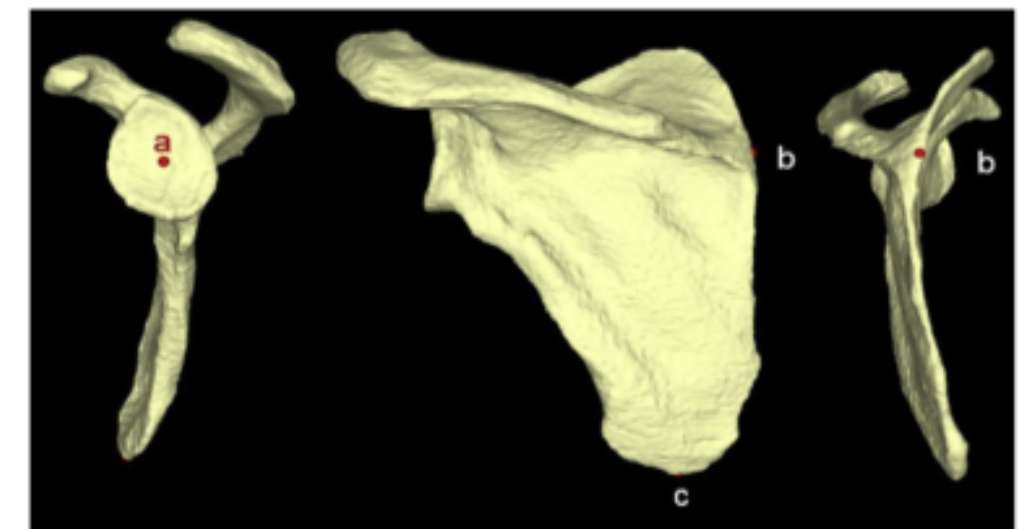

a: Fossa center $(17.7,-19.9,9.8)$

b: Trigonum $(85.9,51.0,30.3)$

c: Inferior angle $(117.3,81.2,-97.8)$
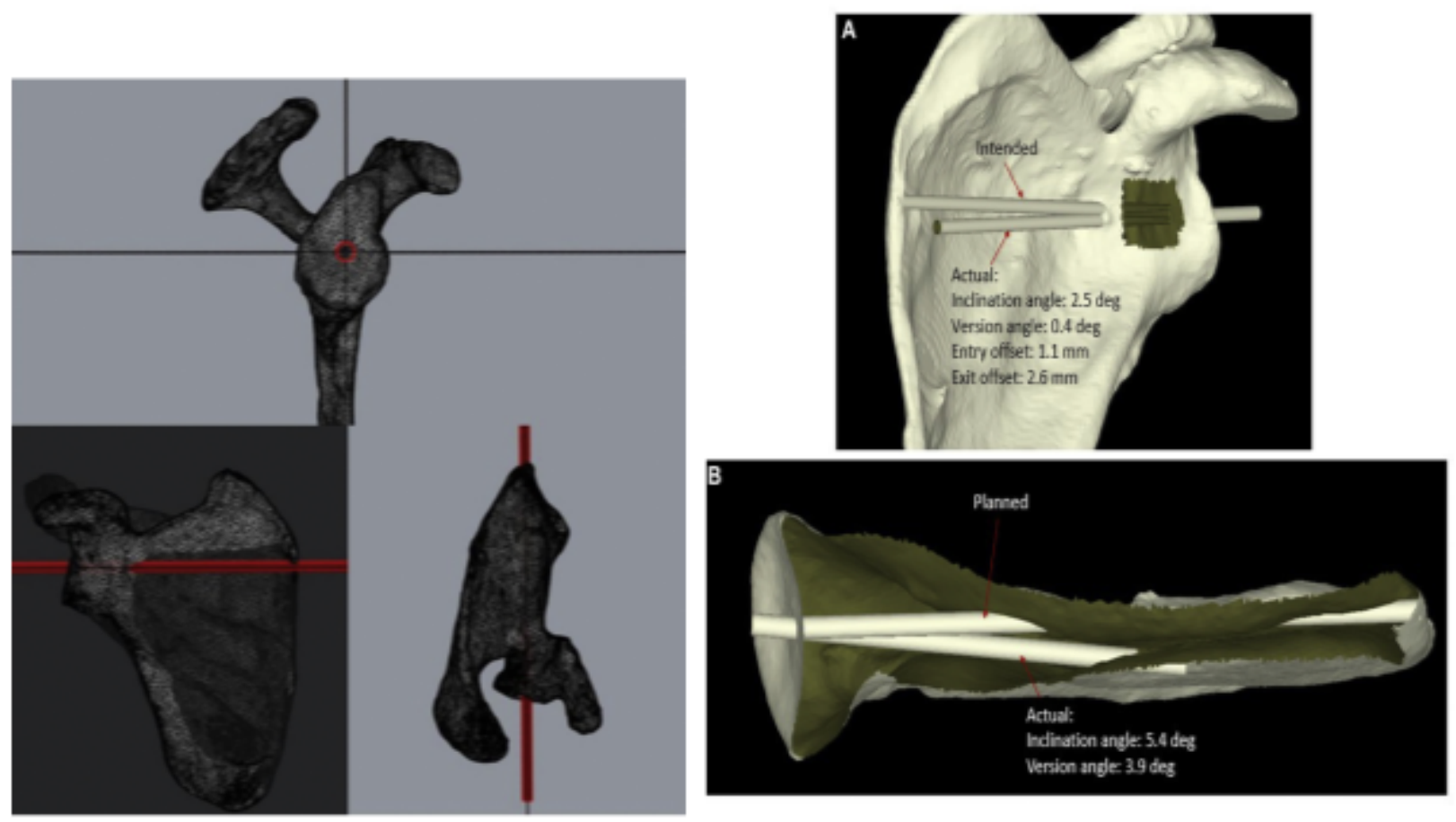

\section{Figure 4}

The STL file was imported into omagics 19.0 to make the support structure and then exported to the magics format of the 3D printing equipment (stereo lithography \sla-450,Shining,China)) to start printing. 

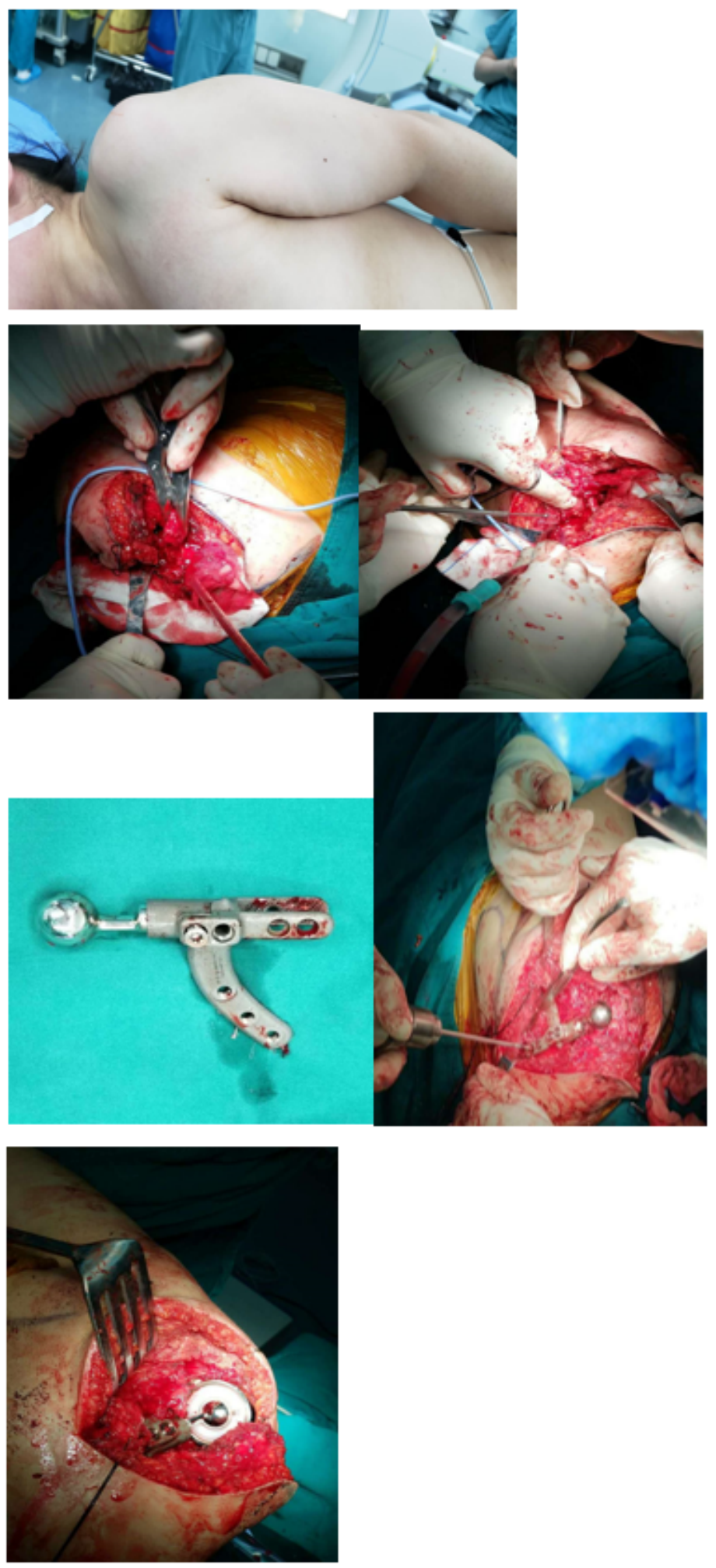

Figure 5

Lateral decubitus position was selected intraoperatively, during the operation, the bone is ground flat on both sides with a drill to ensure that the prosthesis is fully attached to the bone, the reverse shoulder joint prosthesis was successfully implanted 

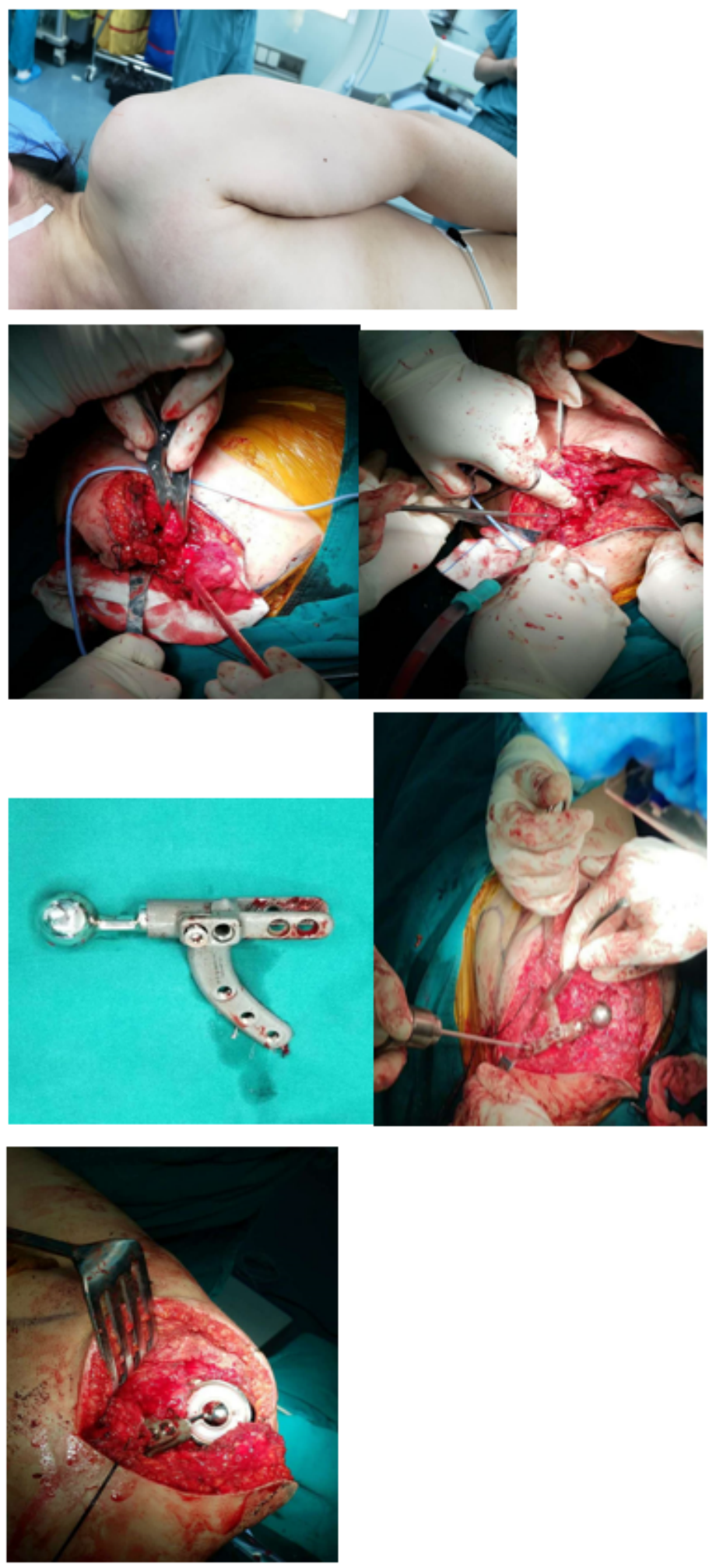

Figure 5

Lateral decubitus position was selected intraoperatively, during the operation, the bone is ground flat on both sides with a drill to ensure that the prosthesis is fully attached to the bone, the reverse shoulder joint prosthesis was successfully implanted 

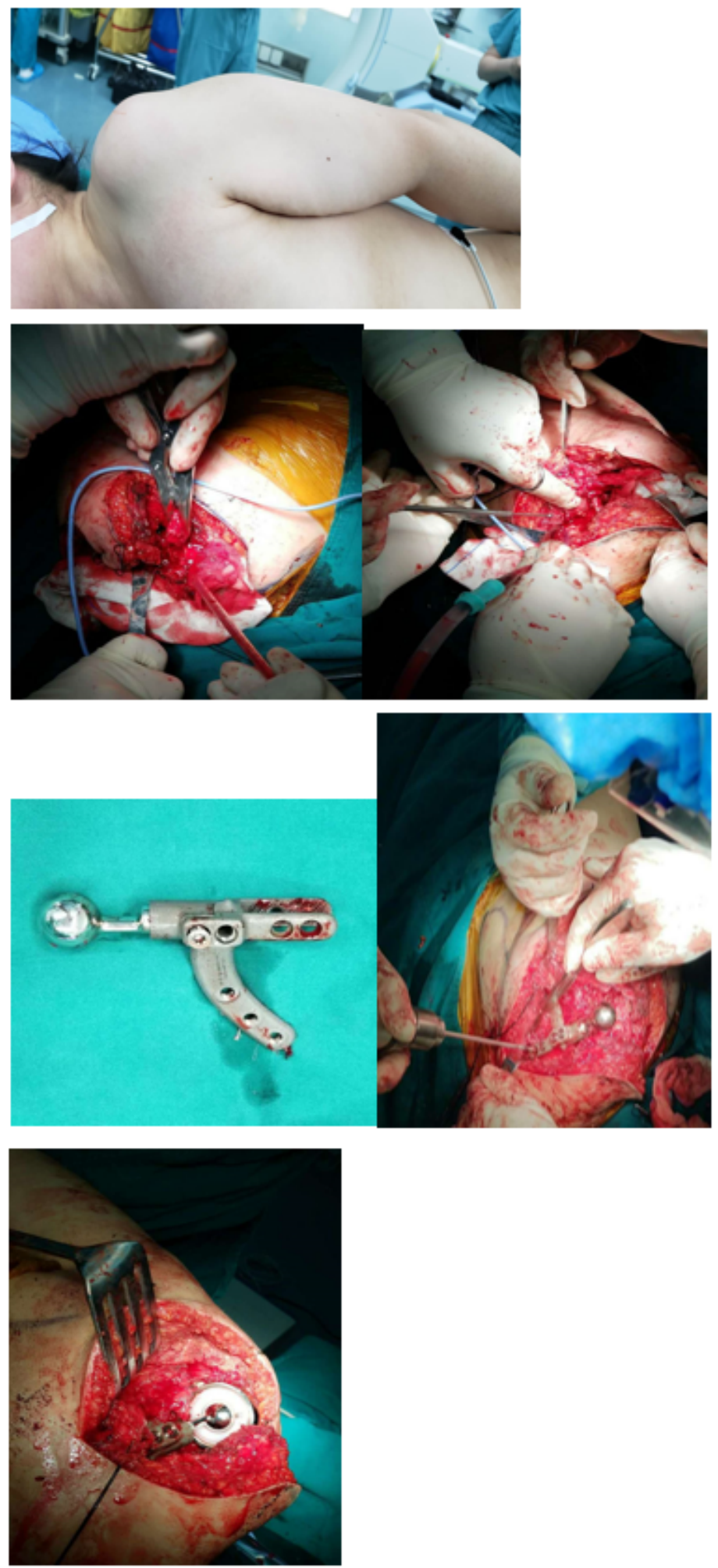

Figure 5

Lateral decubitus position was selected intraoperatively, during the operation, the bone is ground flat on both sides with a drill to ensure that the prosthesis is fully attached to the bone, the reverse shoulder joint prosthesis was successfully implanted 


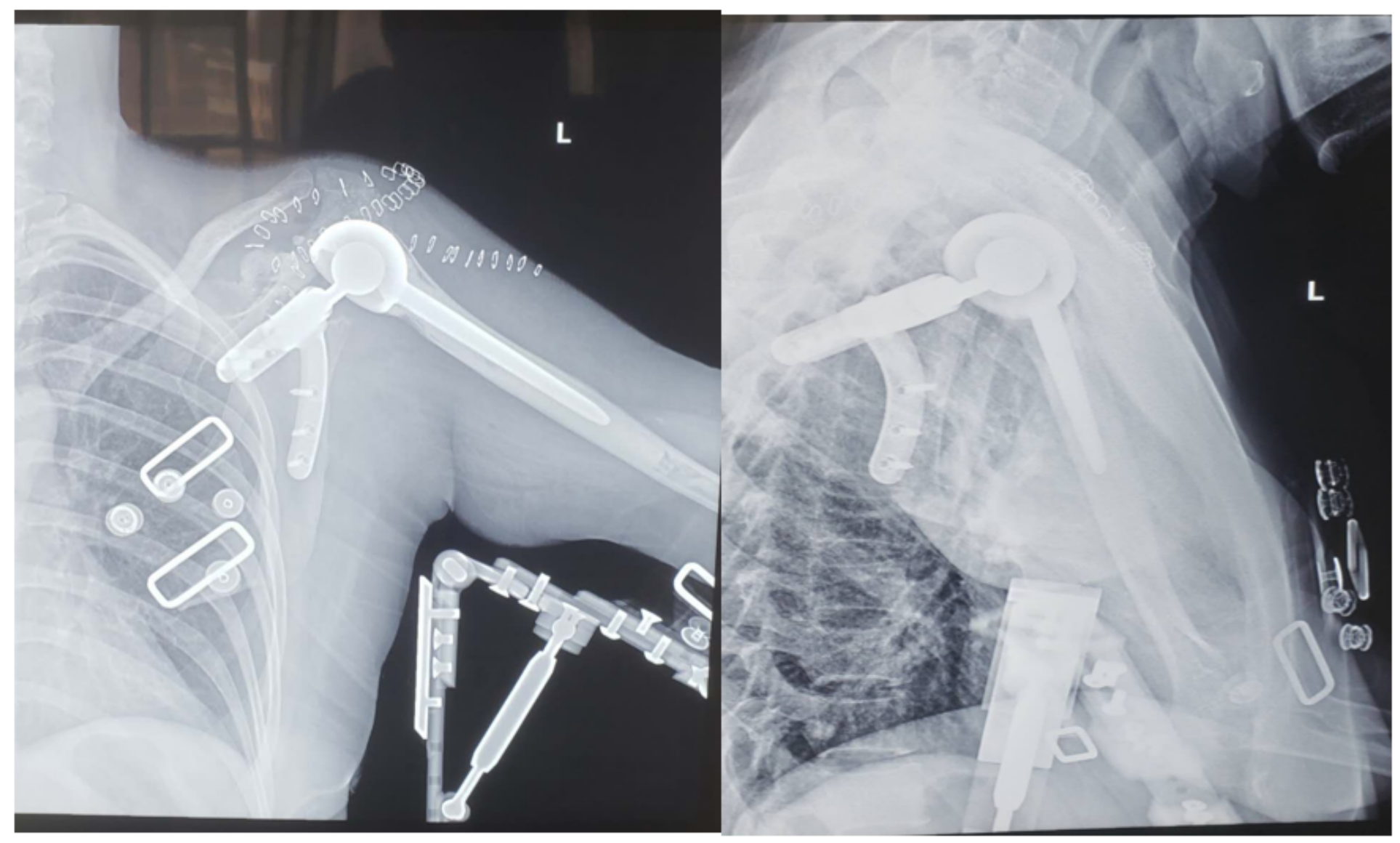

Figure 6

The first day of postoperative X-ray showed that the position of the prosthesis was accurate. 


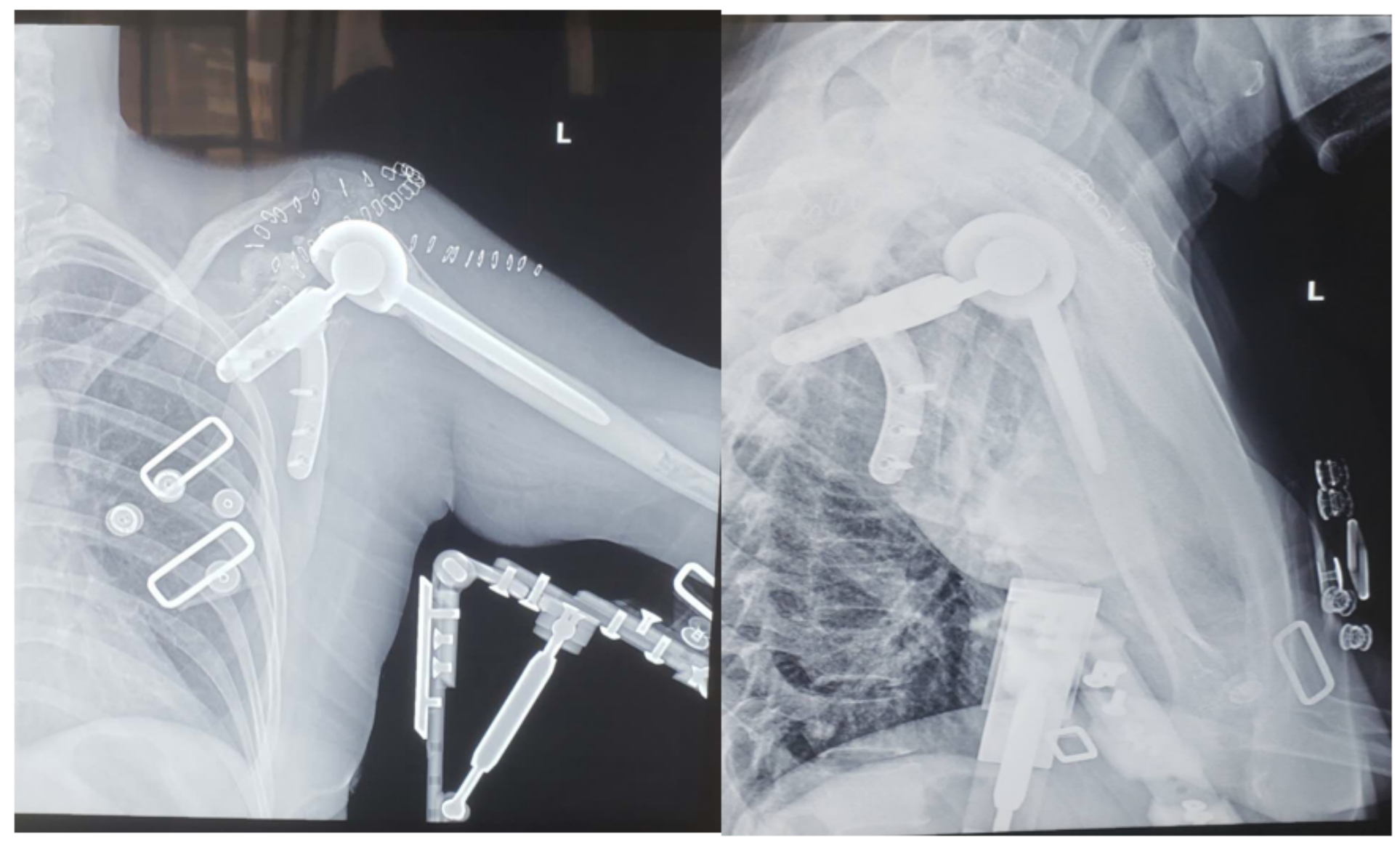

Figure 6

The first day of postoperative X-ray showed that the position of the prosthesis was accurate. 


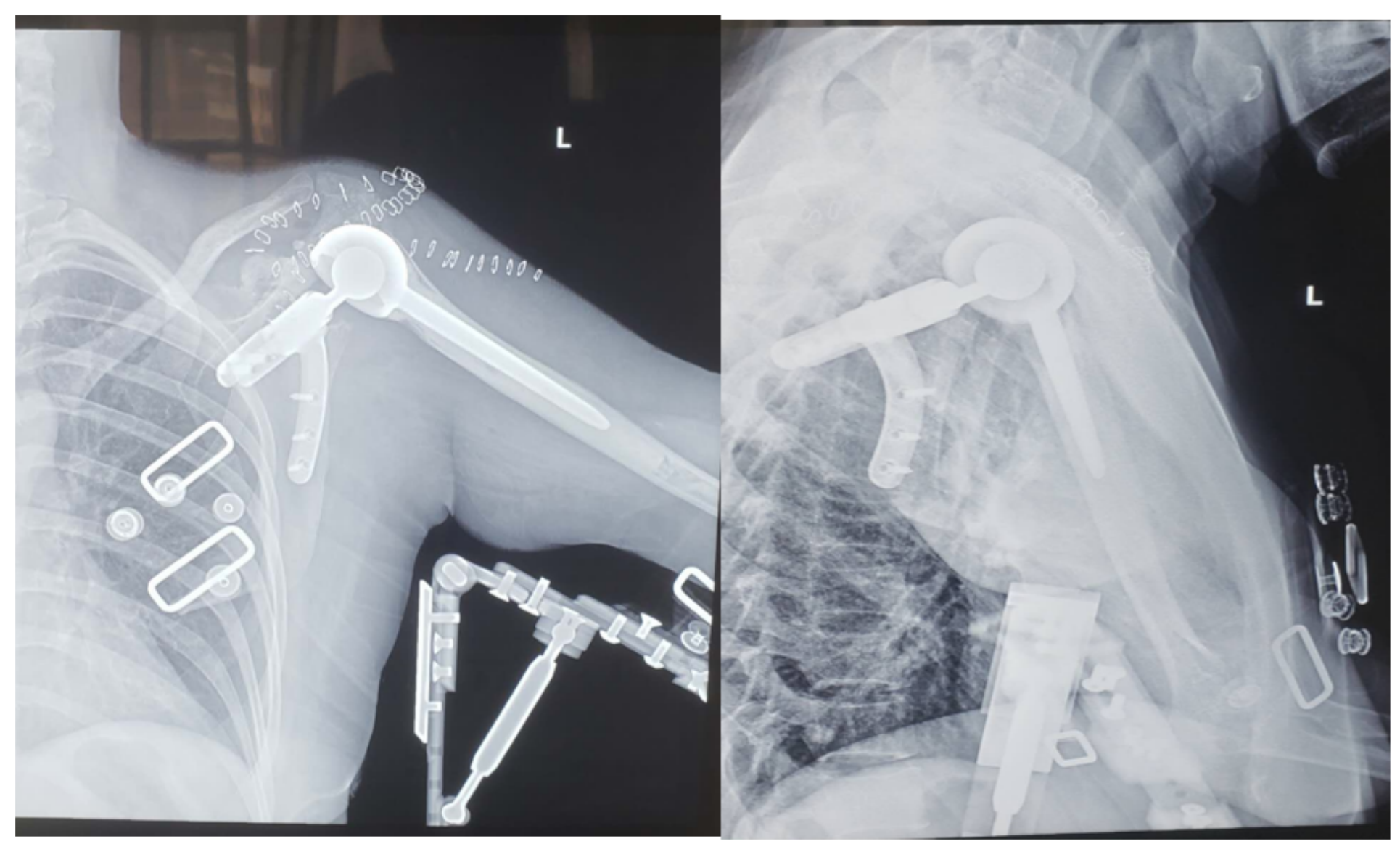

\section{Figure 6}

The first day of postoperative X-ray showed that the position of the prosthesis was accurate.

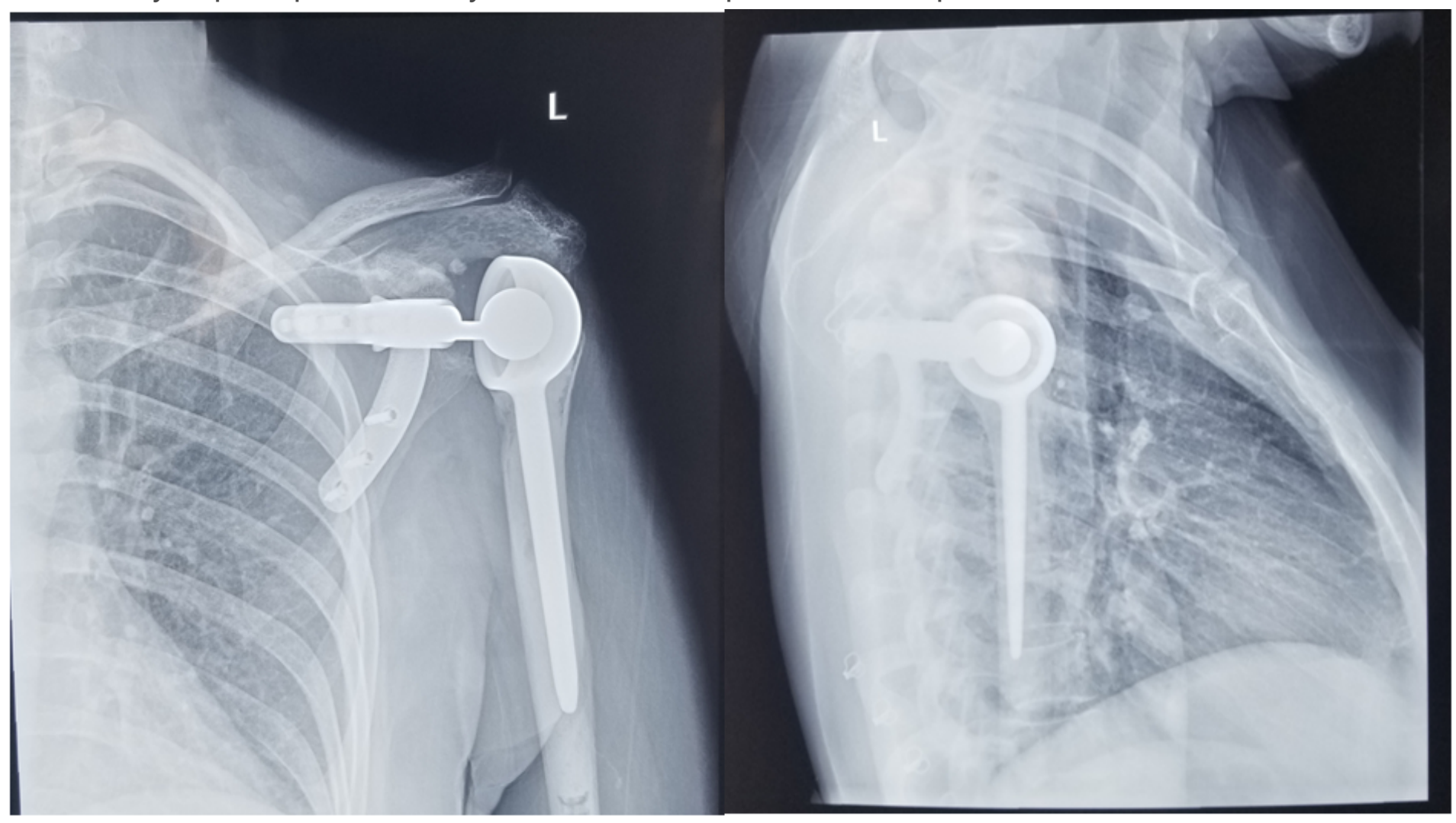


Figure 7

The 3rd month of postoperative X-ray showed that the position of the prosthesis was accurate.

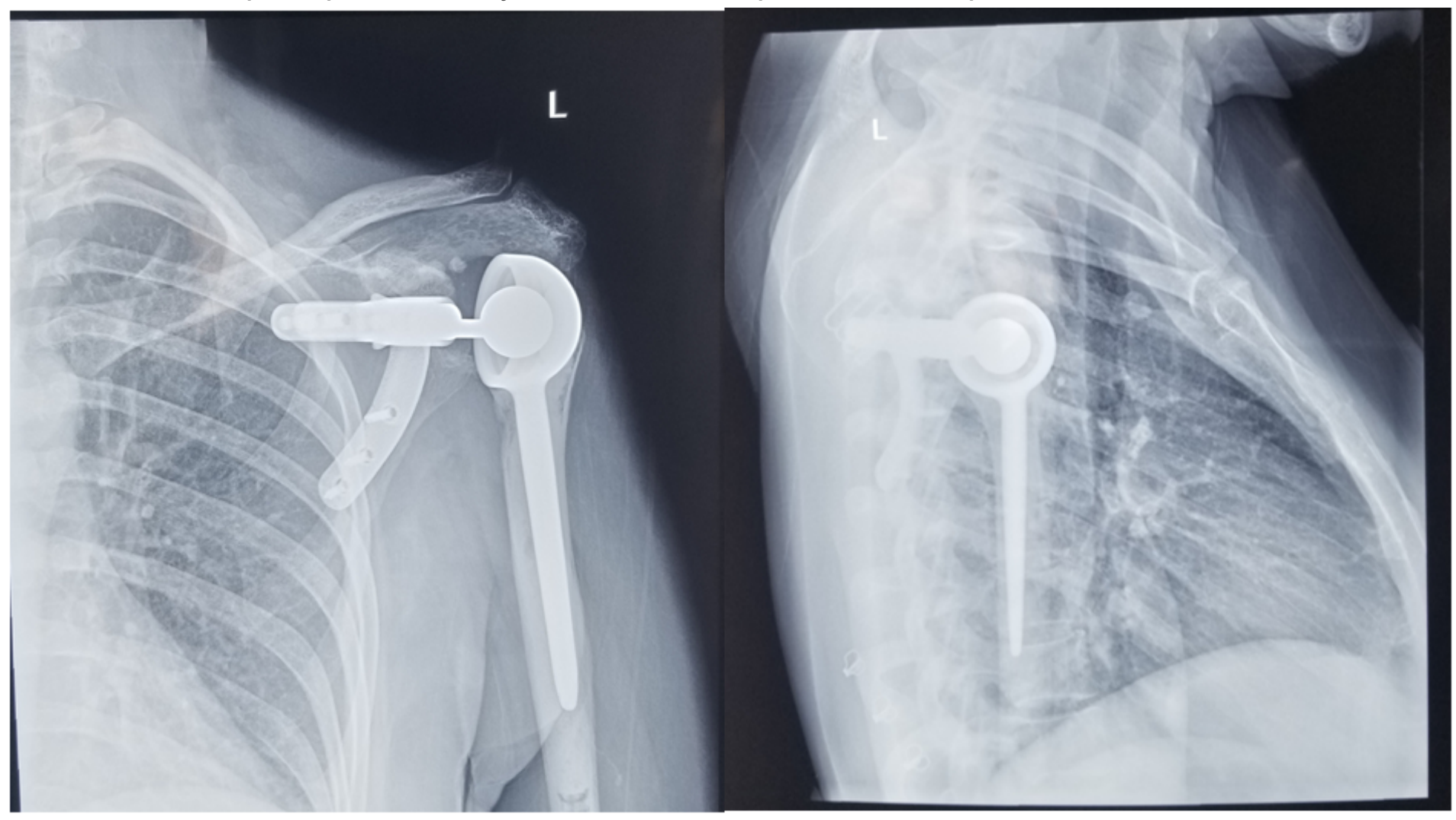

Figure 7

The 3rd month of postoperative X-ray showed that the position of the prosthesis was accurate. 


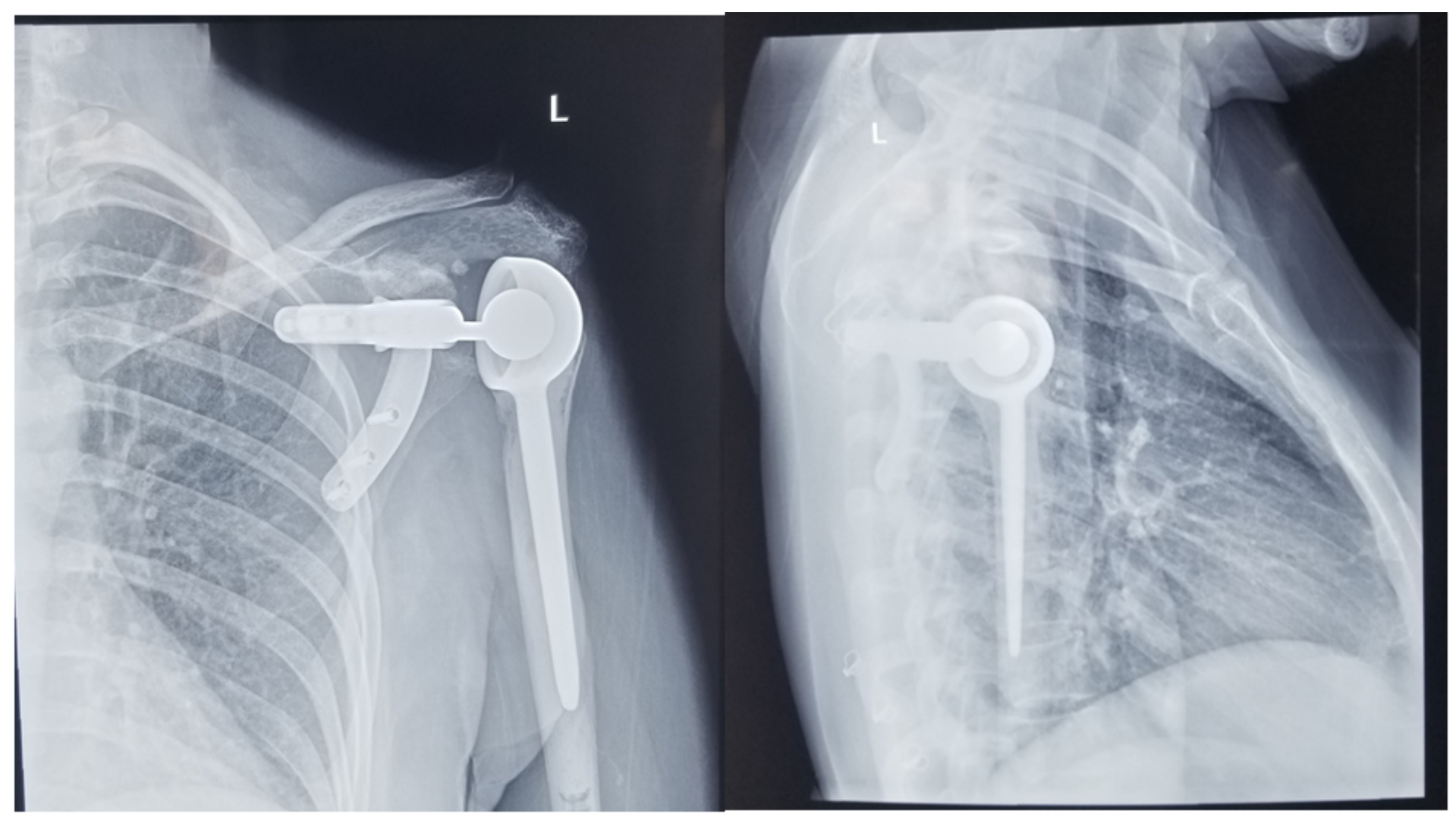

Figure 7

The 3rd month of postoperative X-ray showed that the position of the prosthesis was accurate. 

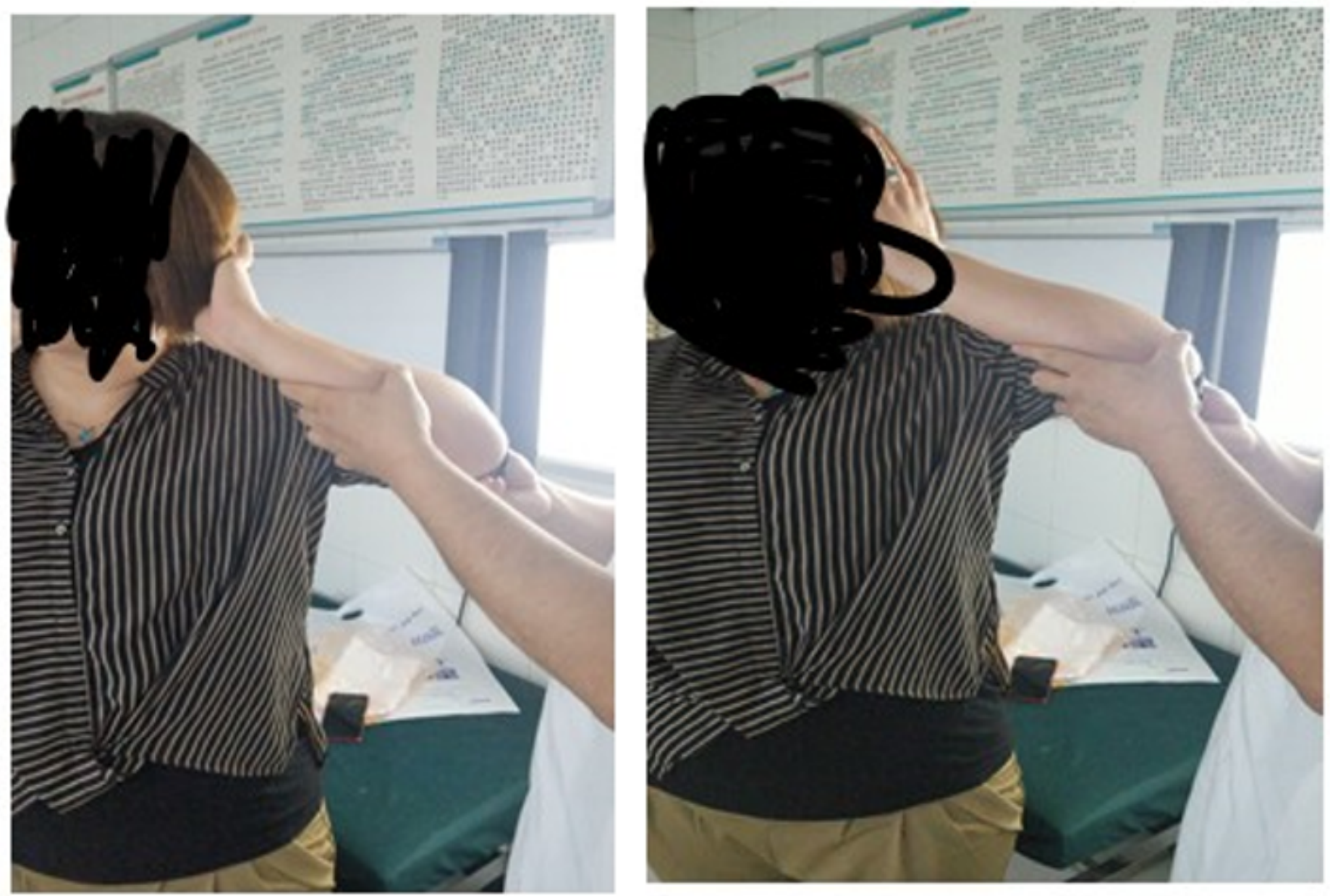

Figure 8

The range of motion (ROM) of shoulder joint was examined at the 7th month. 

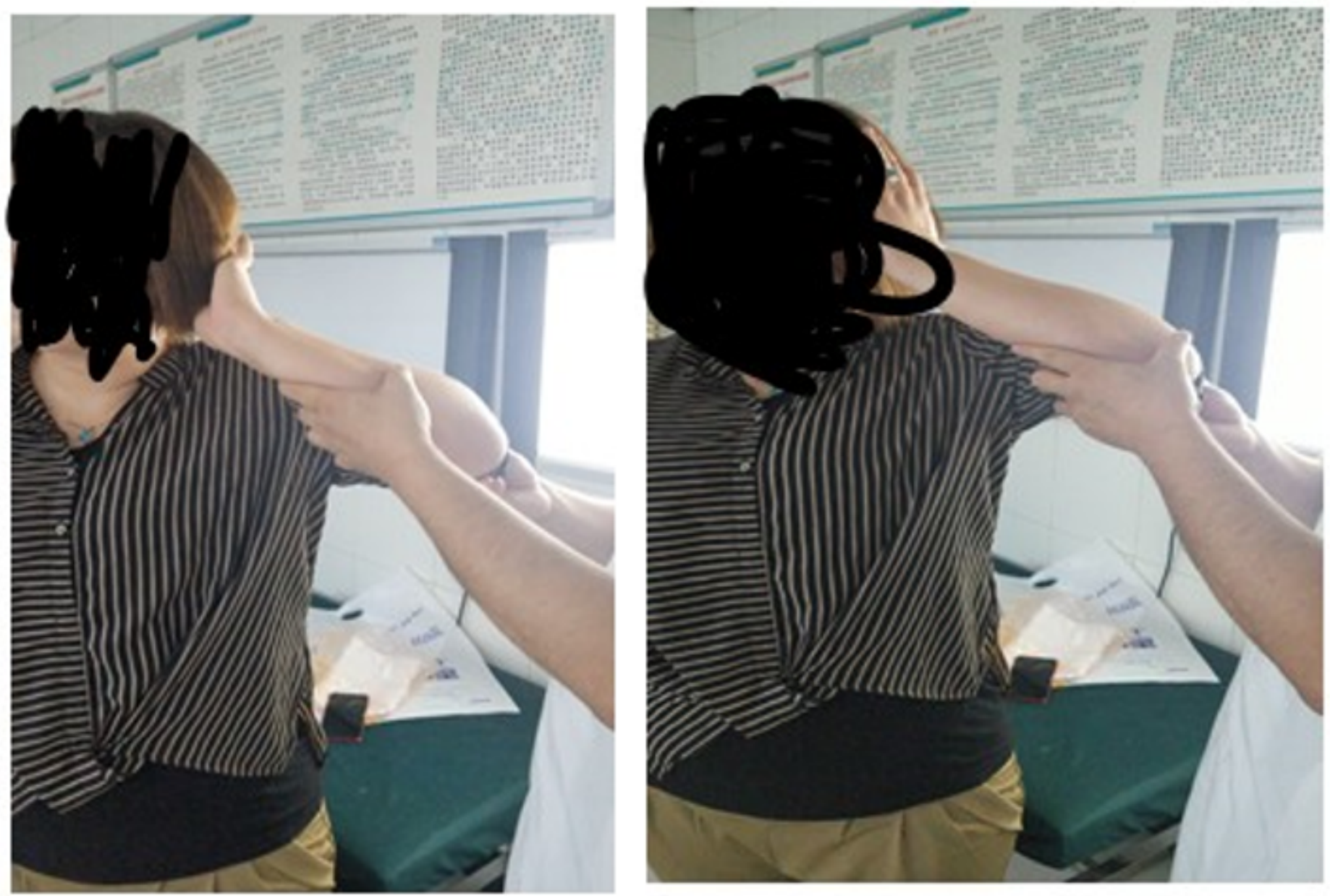

Figure 8

The range of motion (ROM) of shoulder joint was examined at the 7th month. 

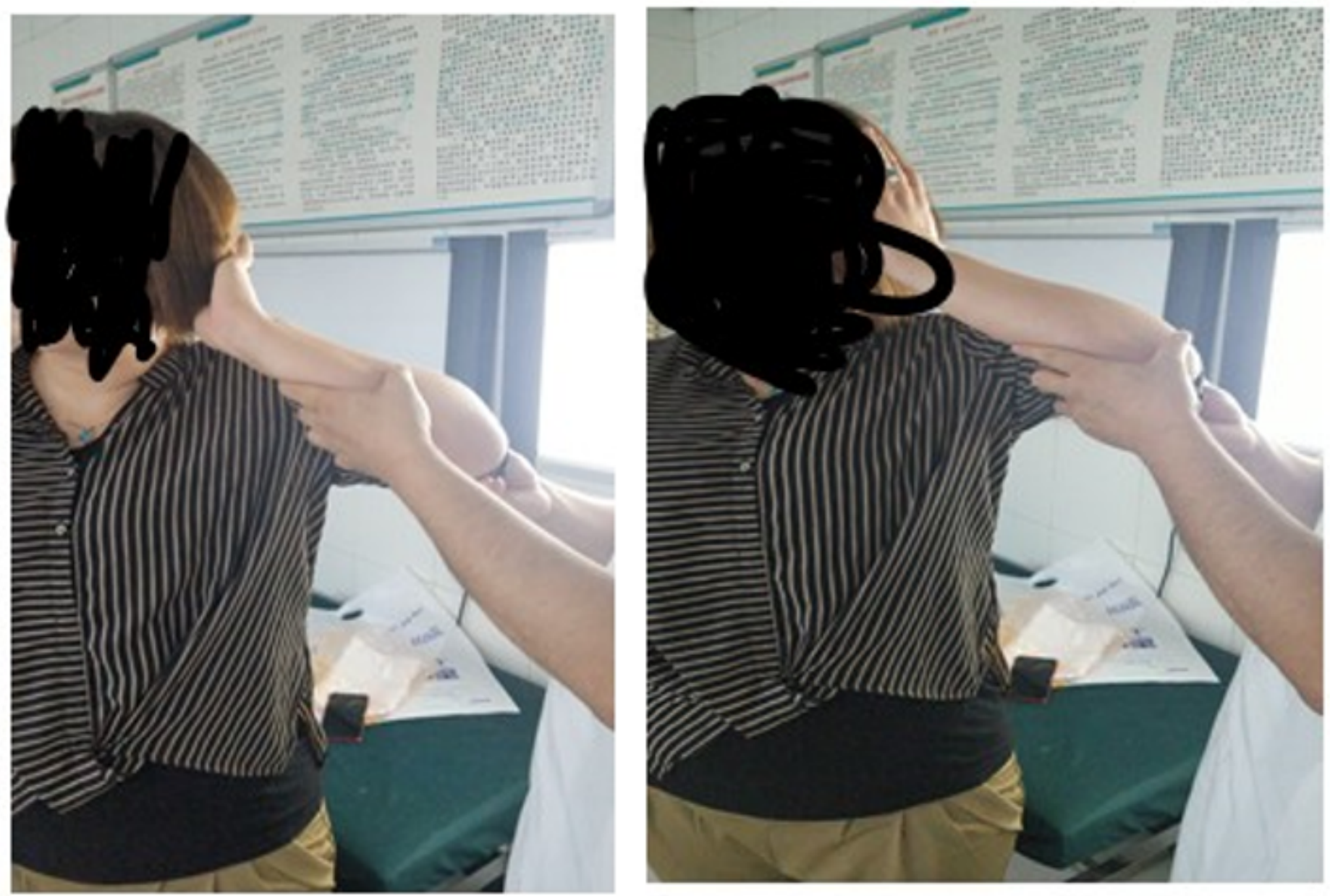

Figure 8

The range of motion (ROM) of shoulder joint was examined at the 7th month. 